\title{
A Higgs Conundrum with Vector Fermions
}

\author{
S. Dawson and E. Furlan \\ Department of Physics, Brookhaven National Laboratory, Upton, NY 11973, USA
}

\begin{abstract}
Many models of Beyond the Standard Model physics involve heavy colored fermions. We study models where the new fermions have vector interactions and examine the connection between electroweak precision measurements and Higgs production. In particular, for parameters which are allowed by precision measurements, we show that the gluon fusion Higgs cross section and the Higgs decay branching ratios must be close to those predicted by the Standard Model. The models we discuss thus represent scenarios with new physics which will be extremely difficult to distinguish from the minimal Standard Model. We pay particular attention to the decoupling properties of the vector fermions.
\end{abstract}




\section{INTRODUCTION}

The Standard Model of particle physics has a remarkable body of experimental support, but the Higgs boson remains a missing ingredient. Precision electroweak measurements suggest that a Standard Model Higgs boson must be lighter than $145 \mathrm{GeV}$ [1, 2] and recent measurements from the LHC exclude a Standard Model Higgs boson in the range $129 \mathrm{GeV}<M_{H}<600 \mathrm{GeV}$ [3]. Preliminary measurements suggest a light Higgs boson in the mass region $M_{H} \sim 125 \mathrm{GeV}$ [3, 4]. Should this putative Higgs signal be confirmed, the pressing issue will be understanding its properties.

For all Higgs masses, gluon fusion is the dominant production mechanism at hadron colliders and the production rate is well understood up to NNLO in QCD [5, 6]. Theoretical uncertainties from renormalization/factorization scale choices and from the choice of parton distribution functions are also well understood [7-10]. The total rate, however, is sensitive to the existence of colored particles which couple to the Higgs boson. Beyond the Standard Model physics can potentially have a large effect on the Higgs boson production rate, making this a window to high scale physics [8, 11 13].

The effects on Higgs production of squarks, Kaluza Klein colored fermions, color octet scalars, fermionic top quark partners and $4^{\text {th }}$ generation fermions (among many others) have been extensively studied. The simplest possibility for new heavy fermions is to form a chiral heavy new generation which, except for masses, is an exact copy of the known generations. After careful tuning, it is possible to find combinations of $4^{\text {th }}$ generation fermion masses which are permitted by precision electroweak measurements [14, 15] and are not excluded by direct searches. Since a chiral $4^{\text {th }}$ generation quark is assumed to couple to the Higgs boson with a strength proportional to its mass, heavy quarks do not decouple from the production of the Higgs boson (and in fact increase the rate by a factor of $\sim 9$ ). the existence of a $4^{\text {th }}$ generation of fermions would exclude a Higgs boson mass up to $M_{H} \sim 600 \mathrm{GeV}$ [16] regardless of the fermion masses.

In this paper, we study the effect of heavy vector quarks on Higgs boson production and study both the case of an isospin singlet top partner and an isospin doublet of heavy fermions. A vector singlet top partner arises naturally in Little Higgs models [17 $[23]$, where the couplings to the Higgs boson of the top quark and its fermion partner are fixed in such a manner as to cancel their quadratically divergent contributions to the Higgs mass 
renormalization. Top-quark fermion partners are also found in top color [24, 25] and top condensate [26-29] models where there is a natural hierarchy of scales such that the top partner obtains a large Dirac mass. Light vector fermions instead typically appear in composite Higgs models [30 33]. Our results are general enough to be applied to any of these models and hence represent a simplification of results which have previously been presented in the context of very specific scenarios.

A study of the $S, T, U$ parameters and the $Z \rightarrow b \bar{b}$ decay rate [34, 35] restricts the allowed parameter space for heavy vector fermions. However, vector fermions have interesting decoupling properties as the mixing with the Standard Model fermions becomes small, which makes a large region of parameter space experimentally viable. Vector fermions which couple to the Standard Model fermions and Higgs boson can be $S U(2)_{L}$ singlets with the same hypercharge as the Standard Model right-handed quarks, doublets with $Y=Y_{S M}=\frac{1}{6}$

or $Y=Y_{S M} \pm 1$, or triplets with $Y=Y_{S M} \pm \frac{1}{2}[36]$. We consider the "Standard Modellike" case with either a heavy fermion singlet of charge $2 / 3$ or a doublet with the Standard Model assignments of hypercharge. We compute the NNLO prediction for Higgs production for the allowed parameter region of these models and quantify the allowed deviation from the Standard Model prediction. The new features of our study include up-to-date fits to precision electroweak measurements in models with vector fermions, and an analysis of the the resulting consequences for Higgs boson production at NNLO in perturbative QCD.

\section{THE MODELS}

We consider models with additional vector-like charge $2 / 3$ quarks, $\mathcal{T}^{\alpha}$, and charge $-1 / 3$ quarks, $\mathcal{B}^{\alpha}$, which mix with the Standard Model-like third generation quarks. For simplicity we make the following assumptions:

- the electroweak gauge group is the standard $S U(2)_{L} \times U(1)_{Y}$ group;

- there is only a single Standard Model Higgs $S U(2)_{L}$ doublet,

$$
H=\left(\begin{array}{l}
\phi^{+} \\
\phi^{0}
\end{array}\right),
$$

with $\phi^{0}=\frac{v+h}{\sqrt{2}}$ 
- we neglect generalized CKM mixing and only allow mixing between the Standard Model-like third generation quarks and at most one new charge $2 / 3$ quark singlet or one new $S U(2)_{L}$ quark doublet. We do not consider fermions in more exotic representations.

The Standard Model-like chiral fermions are

$$
\psi_{L}^{1}=\left(\begin{array}{c}
\mathcal{T}_{L}^{1} \\
\mathcal{B}_{L}^{1}
\end{array}\right), \quad \mathcal{T}_{R}^{1}, \mathcal{B}_{R}^{1}
$$

with the Lagrangian describing the fermion masses

$$
-\mathcal{L}_{M}^{S M}=\lambda_{1} \bar{\psi}_{L}^{1} H \mathcal{B}_{R}^{1}+\lambda_{2} \bar{\psi}_{L}^{1} \tilde{H} \mathcal{T}_{R}^{1}+\text { h.c. }
$$

and $\tilde{H}=i \sigma_{2} H^{*}$.

The models we consider are:

- singlet fermion model: add a vector $S U(2)_{L}$ quark singlet of charge $2 / 3, \mathcal{T}_{L}^{2}$ and $\mathcal{T}_{R}^{2}$.

- doublet fermion model: add a vector $S U(2)_{L}$ doublet of hypercharge $1 / 6$,

$$
\psi_{L}^{2}=\left(\begin{array}{c}
\mathcal{T}_{L}^{2} \\
\mathcal{B}_{L}^{2}
\end{array}\right), \quad \psi_{R}^{2}=\left(\begin{array}{c}
\mathcal{T}_{R}^{2} \\
\mathcal{B}_{R}^{2}
\end{array}\right)
$$

\section{EXPERIMENTAL LIMITS ON TOP PARTNER MODELS}

\section{A. Limits from $R_{b}$ and $A_{b}$}

Data from LEP and SLD place stringent restrictions on the couplings of the fermionic top partners. The top partners mix with the Standard Model-like top quark and contribute at one loop to processes involving bottom quarks, especially $Z \rightarrow b \bar{b}$ and $A_{b}$. The neutral current couplings to the bottom can be parametrized by the effective Lagrangian

$$
\mathcal{L}^{N C}=\frac{g}{c_{W}} Z_{\mu} \bar{f} \gamma^{\mu}\left[\left(g_{L}^{f}+\delta \tilde{g}_{L}^{f}\right)\left(\frac{1-\gamma_{5}}{2}\right)+\left(g_{R}^{f}+\delta \tilde{g}_{R}^{f}\right)\left(\frac{1+\gamma_{5}}{2}\right)\right] f,
$$

where the Standard Model couplings are normalized such that $g_{L}^{f}=T_{3}^{f}-Q_{f} s_{W}^{2}$, $g_{R}^{f}=-Q_{f} s_{W}^{2}$, with $s_{W}^{2} \equiv \sin ^{2} \theta_{W}=(e / g)^{2}=0.231$ [37] and $T_{3}^{f}= \pm 1 / 2$. The couplings

$\delta \tilde{g}_{L, R}^{f} \equiv \delta g_{L, R}^{f, S M}+\delta g_{L, R}^{f}$ contain both the Standard Model radiative corrections, $\delta g_{L, R}^{f, S M}$, and 
95\% Confidence Level Limits

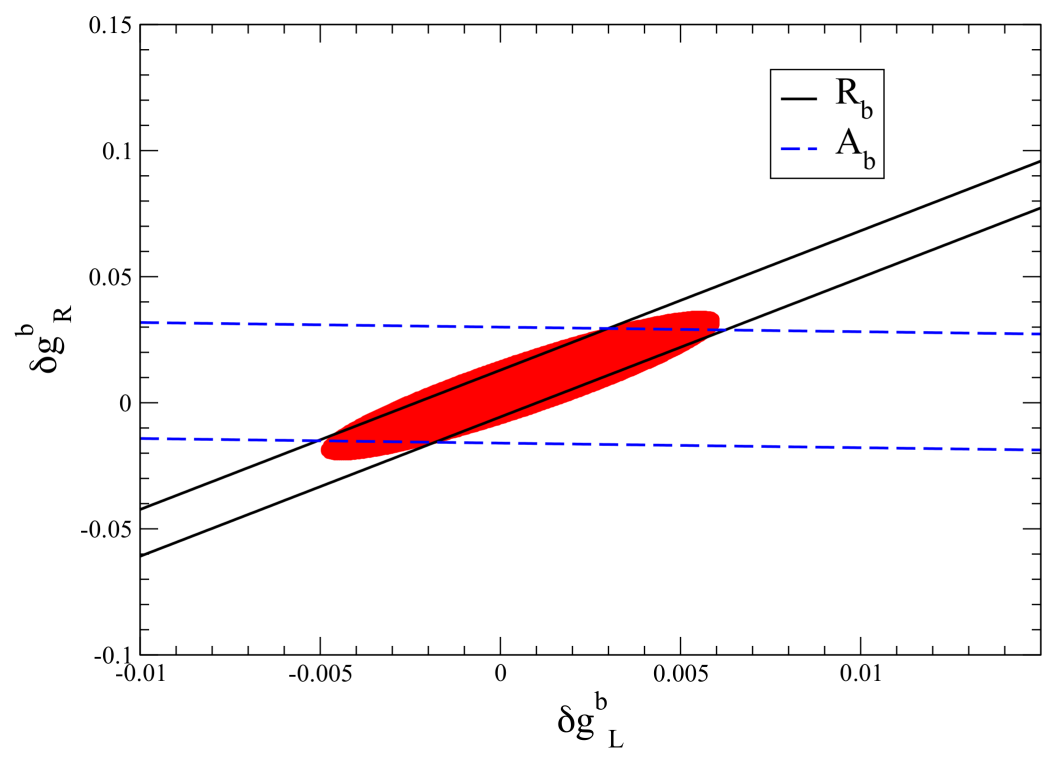

FIG. 1: Allowed 95\% confidence level regions from the simultaneous fit to $R_{b}$ and $A_{b}$ (red shaded), $R_{b}$ alone (between solid black lines), and $A_{b}$ alone (between dashed blue lines).

the new physics contributions, $\delta g_{L, R}^{f}$. The Standard Model contribution from top quark loops is well known [38-40], and in the limit $m_{t}>>M_{Z}$ it is given by

$$
\delta g_{L}^{b, S M}=\frac{G_{F}}{\sqrt{2}} \frac{m_{t}^{2}}{8 \pi^{2}} .
$$

The dominant effect of new physics in the $b$ sector can be found by assuming that $\delta g_{L}^{b}$ and $\delta g_{R}^{b}$ are small and approximating [41, 42]

$$
\begin{aligned}
& R_{b}=\frac{\Gamma(Z \rightarrow b \bar{b})}{\Gamma(Z \rightarrow h a d r o n s)}=R_{b}^{S M}\left\{1-3.57 \delta g_{L}^{b}+0.65 \delta g_{R}^{b}\right\} \\
& A_{b}=\frac{\left(\delta \tilde{g}_{L}^{b}\right)^{2}-\left(\delta \tilde{g}_{R}^{b}\right)^{2}}{\left(\delta \tilde{g}_{L}^{b}\right)^{2}+\left(\delta \tilde{g}_{R}^{b}\right)^{2}}=A_{b}^{S M}\left\{1-0.31 \delta g_{L}^{b}-1.72 \delta g_{R}^{b}\right\}
\end{aligned}
$$

where $R_{b}^{S M}$ and $A_{b}^{S M}$ are the theory predictions including all radiative corrections. The positive contribution to $\delta g_{L}^{b, S M}$ from the top quark has the effect of reducing both $R_{b}^{S M}$ and $A_{b}^{S M}$.

The 95\% confidence level ellipse for new $Z b \bar{b}$ couplings is shown in Fig. 1 and is obtained 
using the Particle Data Group results [37, 43]

$$
\begin{aligned}
& R_{b}^{e x p}=0.21629 \pm 0.00066 \\
& R_{b}^{S M}=0.21578 \pm 0.00005 \\
& A_{b}^{\text {exp }}=0.923 \pm 0.020 \\
& A_{b}^{S M}=0.9348 \pm 0.0001 .
\end{aligned}
$$

If $\delta g_{R}^{b}=0$, the $95 \%$ confidence level limit from the fit to $A_{b}$ and $R_{b}$ is

$$
-0.0027<\delta g_{L}^{b}<0.0014
$$

Similarly, if $\delta g_{L}^{b}=0$, the $95 \%$ confidence level limit from the fit to $A_{b}$ and $R_{b}$ is

$$
-0.0066<\delta g_{R}^{b}<0.0148
$$

\section{B. Limits from the Oblique Parameters $S, T$ and $U$}

The new quarks contribute at loop level to the vacuum polarizations of the electroweak gauge bosons $\Pi_{X Y}^{\mu \nu}\left(p^{2}\right)=\Pi_{X Y}\left(p^{2}\right) g^{\mu \nu}+B_{X Y}\left(p^{2}\right) p^{\mu} p^{\nu}$, with $X Y=\gamma \gamma, \gamma Z, Z Z$ and $W^{+} W^{-}$[44, 45]. These effects can be parametrized using the $S, T$ and $U$ functions of Peskin and Takeuchi [44],

$$
\begin{aligned}
\alpha S_{F}= & \frac{4 s_{W}^{2} c_{W}^{2}}{M_{Z}^{2}}\left\{\Pi_{Z Z}\left(M_{Z}^{2}\right)-\Pi_{Z Z}(0)-\Pi_{\gamma \gamma}\left(M_{Z}^{2}\right)-\frac{c_{W}^{2}-s_{W}^{2}}{c_{W} s_{W}} \Pi_{\gamma Z}\left(M_{Z}^{2}\right)\right\} \\
\alpha T_{F}= & \frac{\Pi_{W W}(0)}{M_{W}^{2}}-\frac{\Pi_{Z Z}(0)}{M_{Z}^{2}} \\
\alpha U_{F}= & 4 s_{W}^{2}\left\{\frac{\Pi_{W W}\left(M_{W}^{2}\right)-\Pi_{W W}(0)}{M_{W}^{2}}-c_{W}^{2}\left(\frac{\Pi_{Z Z}\left(M_{Z}^{2}\right)-\Pi_{Z Z}(0)}{M_{Z}^{2}}\right)\right. \\
& \left.-2 s_{W} c_{W} \frac{\Pi_{\gamma Z}\left(M_{Z}^{2}\right)}{M_{Z}^{2}}-s_{W}^{2} \frac{\Pi_{\gamma \gamma}\left(M_{Z}^{2}\right)}{M_{Z}^{2}}\right\} .
\end{aligned}
$$

Any definition of $s_{W}$ can be used in Eq. 11 since the scheme dependence enters at higher

order. Since these parameters are well-constrained by LEP and LEP2 measurements [46], they set stringent limits on the masses and couplings of the new quarks.

We use the fit to the electroweak precision data given in Refs. [2, 47],

$$
\begin{gathered}
\Delta S=S-S_{S M}=0.02 \pm 0.11 \\
\Delta T=T-T_{S M}=0.05 \pm 0.12 \\
\Delta U=U-U_{S M}=0.07 \pm 0.12
\end{gathered}
$$


with reference Higgs and top-quark masses $M_{H, \text { ref }}=120 \mathrm{GeV}$ and $m_{t, \text { ref }}=173.1 \mathrm{GeV}$. The associated correlation matrix is

$$
\rho_{i j}=\left(\begin{array}{lll}
1.0 & 0.879 & -0.469 \\
0.879 & 1.0 & -0.716 \\
-0.469 & -0.716 & 1.0
\end{array}\right) .
$$

The $\Delta \chi^{2}$ is defined as

$$
\Delta \chi^{2}=\sum_{i, j}\left(\Delta X_{i}-\Delta \hat{X}_{i}\right)\left(\sigma^{2}\right)_{i j}^{-1}\left(\Delta X_{j}-\Delta \hat{X}_{j}\right)
$$

where $\Delta \hat{X}_{i}=\Delta S, \Delta T$ and $\Delta U$ are the central values of the fit in Eq. 12, $\Delta X_{i}=X_{i}-X_{i}^{S M}=$ $\Delta S_{F}, \Delta T_{F}$ and $\Delta U_{F}$ are the contributions to the oblique parameters from the new fermions and $\sigma_{i j}^{2} \equiv \sigma_{i} \rho_{i j} \sigma_{j}, \sigma_{i}$ being the errors given in Eq. 12, A $95 \%$ confidence level limit in a three-parameter fit corresponds to $\Delta \chi^{2}=7.82$.

Since we consider primarily $M_{H}=125 \mathrm{GeV}$, we need to add the Higgs contributions ${ }^{1}$

$$
\begin{aligned}
\Delta S_{H} & =\frac{1}{12 \pi} \log \left(\frac{M_{H}^{2}}{M_{H, \text { ref }}^{2}}\right)+\mathcal{O}\left(\frac{M_{Z}^{2}}{M_{H}^{2}}\right) \\
\Delta T_{H} & =-\frac{3}{16 \pi c_{W}^{2}} \log \left(\frac{M_{H}^{2}}{M_{H, \text { ref }}^{2}}\right)+\mathcal{O}\left(\frac{M_{Z}^{2}}{M_{H}^{2}}\right) \\
\Delta U_{H} & =\mathcal{O}\left(\frac{M_{Z}^{2}}{M_{H}^{2}}\right) .
\end{aligned}
$$

\section{Other Experimental Limits on Top Partner Models}

Both ATLAS [50, 51] and CMS [52, 53] have searched for direct pair production of new heavy fermions. For charge $2 / 3$ top-like quarks decaying with $100 \%$ branching ratio to $W b$, CMS excludes masses below $557 \mathrm{GeV}$ at $95 \%$ confidence level, while ATLAS sets an upper bound of $404 \mathrm{GeV}$. CMS dedicates a specific analysis to pair-produced vector quarks of charge $2 / 3$ decaying entirely to $Z t$, excluding masses below $475 \mathrm{GeV}[54]$. For charge $-1 / 3$ quarks, assuming $100 \%$ branching ratio to $Z b$, ATLAS excludes masses below $358 \mathrm{GeV}$ for a vector singlet, while CMS excludes charge $-1 / 3$ quarks decaying with $100 \%$ branching ratio to $W t$ below $611 \mathrm{GeV}$. These limits are not directly applicable to our models, since the

\footnotetext{
${ }^{1}$ Our fits include the exact results for the Higgs contributions, which can be found in many places including Ref. [48] and the Appendix of Ref. [49].
} 
branching ratios of the new heavy fermions to Standard Model particles are degraded by mixing angles and the limits therefore weakened [36, 55 -59]. Our results on Higgs production are rather insensitive to the masses of the new top partners and we typically assume masses of the TeV scale.

In principle, there are also limits on heavy charged fermions which mix with the Standard Model third generation quarks coming from $K, B$ and $D$ rare processes. For TeV-scale masses of the new fermions and small mixing parameters (which we will see in the next section are required by limits from oblique parameters, $R_{b}$ and $A_{b}$ ), the constraints from rare processes are not restrictive [57, 59 61].

\section{SINGLET TOP PARTNER MODEL}

Little Higgs models [17-23], topcolor models [24, 25] and top condensate models [26-28] all contain a charge $2 / 3$ partner of the top quark, which we denote by $\mathcal{T}^{2}$. We consider a general case with a vector $S U(2)_{L}$ singlet fermion which is allowed to mix with the Standard Model-like top quark [19, 36, 60, 62, 63]. The mass eigenstates are $b \equiv \tilde{\mathcal{B}}^{1}, t$ and $T$, where $b$ and $t$ are the observed bottom and top quarks. Thorough this paper we will use the measured mass values $m_{b}=4.19 \mathrm{GeV}, m_{t}=173.1 \mathrm{GeV}$ [37, 64]. The mass eigenstates of charge $2 / 3$ can be found through the rotations

$$
\chi_{L, R}^{t} \equiv\left(\begin{array}{c}
t_{L, R} \\
T_{L, R}
\end{array}\right) \equiv U_{L, R}^{t}\left(\begin{array}{c}
\mathcal{T}_{L, R}^{1} \\
\mathcal{T}_{L, R}^{2}
\end{array}\right)
$$

The matrices $U_{L, R}^{t}$ are unitary and $\Psi_{L, R} \equiv \frac{1 \mp \gamma_{5}}{2} \Psi$. The mixing matrices are parametrized as

$$
U_{L}^{t}=\left(\begin{array}{cc}
\cos \theta_{L} & -\sin \theta_{L} \\
\sin \theta_{L} & \cos \theta_{L}
\end{array}\right), \quad U_{R}^{t}=\left(\begin{array}{cc}
\cos \theta_{R} & -\sin \theta_{R} \\
\sin \theta_{R} & \cos \theta_{R}
\end{array}\right) .
$$

We abbreviate $c_{L} \equiv \cos \theta_{L}, s_{L} \equiv \sin \theta_{L}$.

The most general fermion mass terms are

$$
\begin{aligned}
-\mathcal{L}_{M, 1} & =-\mathcal{L}_{M}^{S M}+\lambda_{3} \bar{\psi}_{L}^{1} \tilde{H} \mathcal{T}_{R}^{2}+\lambda_{4} \overline{\mathcal{T}}_{L}^{2} \mathcal{T}_{R}^{1}+\lambda_{5} \overline{\mathcal{T}}_{L}^{2} \mathcal{T}_{R}^{2}+\text { h.c. } \\
& =\bar{\chi}_{L}^{t}\left[U_{L}^{t}\left(M_{(1)}^{t}+h H_{(1)}^{t}\right) U_{R}^{t, \dagger}\right] \chi_{R}^{t}+\lambda_{1} \frac{v+h}{\sqrt{2}} \overline{\mathcal{B}}_{L}^{1} \mathcal{B}_{R}^{1}+\text { h.c. }
\end{aligned}
$$

where

$$
M_{(1)}^{t}=\left(\begin{array}{cc}
\lambda_{2} \frac{v}{\sqrt{2}} & \lambda_{3} \frac{v}{\sqrt{2}} \\
\lambda_{4} & \lambda_{5}
\end{array}\right) \quad, \quad H_{(1)}^{t}=\frac{1}{\sqrt{2}}\left(\begin{array}{cc}
\lambda_{2} & \lambda_{3} \\
0 & 0
\end{array}\right)
$$


The resulting mass eigenstates are

$$
\mathcal{M}^{t, \operatorname{diag}} \equiv\left(\begin{array}{cc}
m_{t} & 0 \\
0 & M_{T}
\end{array}\right)
$$

One can always rotate $\mathcal{T}^{2}$ such that $\lambda_{4}=0$. Since $\lambda_{4}$ can be rotated away, the model has four free parameters. Alternatively, it is always possible to rotate $\mathcal{T}_{R}^{2}$ such that $\sin \theta_{R}=0$, because only the Standard Model-like left-handed doublet $\psi_{L}^{1}$ mixes to the singlet with a Yukawa term ${ }^{2}$. Therefore the couplings only depend on $\theta_{L}$, which we will take as one of the four physical parameters along with $m_{b}$ (physical mass of the charge $-1 / 3$ quark), $m_{t}$ and $M_{T}$ (physical masses of the charge $2 / 3$ quarks).

The physical masses and mixing angles are found using bi-unitary transformations,

$$
\begin{aligned}
\left(\mathcal{M}^{t, \text { diag }}\right)^{2} & =U_{L}^{t} M_{(1)}^{t} M_{(1)}^{t, \dagger} U_{L}^{t, \dagger} \\
& =U_{R}^{t} M_{(1)}^{t, \dagger} M_{(1)}^{t} U_{R}^{t, \dagger}
\end{aligned}
$$

It is straightforward to find the mass eigenstates and mixing angles,

$$
\begin{aligned}
\tan \left(2 \theta_{R}\right) & =\frac{2 \lambda_{4} \lambda_{5}+v^{2} \lambda_{2} \lambda_{3}}{\lambda_{5}^{2}-\lambda_{4}^{2}+\frac{v^{2}}{2}\left(\lambda_{3}^{2}-\lambda_{2}^{2}\right)} \\
\tan \left(2 \theta_{L}\right) & =\frac{\sqrt{2} v\left(\lambda_{2} \lambda_{4}+\lambda_{3} \lambda_{5}\right)}{\lambda_{5}^{2}+\lambda_{4}^{2}-\frac{v^{2}}{2}\left(\lambda_{2}^{2}+\lambda_{3}^{2}\right)} \\
m_{t} M_{T} & =\frac{v}{\sqrt{2}}\left|\lambda_{2} \lambda_{5}-\lambda_{3} \lambda_{4}\right| \\
M_{T}^{2}+m_{t}^{2} & =\frac{v^{2}}{2}\left(\lambda_{2}^{2}+\lambda_{3}^{2}\right)+\lambda_{4}^{2}+\lambda_{5}^{2} .
\end{aligned}
$$

From Eq. 17, the couplings to the Higgs boson are

$$
\mathcal{L}_{1}^{h}=-\frac{m_{t}}{v} c_{t t} \bar{t}_{L} t_{R} h-\frac{M_{t}}{v} c_{T T} \bar{T}_{L} T_{R} h-\frac{M_{t}}{v} c_{t T} \bar{t}_{L} T_{R} h-\frac{m_{t}}{v} c_{T t} \bar{T}_{L} t_{R} h+\text { h.c. },
$$

where

$$
c_{t t}=c_{L}^{2}, \quad c_{T T}=s_{L}^{2}, \quad c_{t T}=c_{T t}=s_{L} c_{L} .
$$

These relations can be easily derived by noticing that

$$
v H_{(1), k s}^{t}=M_{(1), k s}^{t} \delta_{k 1}
$$

2 We do not perform any of these rotations here, and the formulas in this section hold for the arbitrary Yukawa couplings of Eq. 17. 
yielding for the physical Higgs couplings

$$
\begin{aligned}
H_{i j} & \equiv U_{L, i k}^{t} H_{(1), k s}^{t} U_{R, s j}^{t, \dagger} \\
& =v^{-1} U_{L, i \hat{k}}^{t} \delta_{\hat{k} 1}\left[U_{L, \hat{k} r}^{t, \dagger} \mathcal{M}_{r r}^{t, d i a g} U_{R, r s}^{t}\right] U_{R, s j}^{t, \dagger} \\
& =v^{-1} U_{L, \hat{k} \hat{k}}^{t} \delta_{\hat{k} 1} U_{L, \hat{k} j}^{t, \dagger} \mathcal{M}_{j j}^{t, d i a g},
\end{aligned}
$$

where the index $\hat{k}$ is not summed over.

The charged and neutral current interactions are

$$
\mathcal{L}_{1}^{C C}=\frac{g}{\sqrt{2}} W^{\mu+} \sum_{i=1,2}\left(\bar{\chi}_{L}^{t}\right)_{i}\left(U_{L}^{t}\right)_{i, 1} \gamma_{\mu} b_{L}+\text { h.c. }
$$

and

$$
\begin{aligned}
\mathcal{L}_{1}^{N C}= & \frac{g}{c_{W}} Z_{\mu} \sum_{i=t, T}\left\{\overline{f_{i}} \gamma^{\mu}\left[\left(g_{L}^{i}+\delta \tilde{g}_{L}^{i}\right) P_{L}+\left(g_{R}^{i}+\delta \tilde{g}_{R}^{i}\right) P_{R}\right] f_{i}\right\} \\
& +\frac{g}{c_{W}} Z_{\mu} \sum_{i \neq j}\left\{\overline{f_{i}} \gamma^{\mu}\left[\delta g_{L}^{i j} P_{L}+\delta g_{R}^{i j} P_{R}\right] f_{j}\right\}
\end{aligned}
$$

where $\delta \tilde{g}_{L, R}^{i}=\delta g_{L, R}^{i, S M}+\delta g_{L, R}^{i}$ contains both the Standard Model contribution from top quark loops and the new physics contributions. The new physics couplings arising from the interactions of the top partner singlet are

$$
\begin{array}{ll}
\delta g_{L}^{t}=-\frac{s_{L}^{2}}{2} \quad, \quad \delta g_{L}^{T}=-\frac{c_{L}^{2}}{2} \quad, \quad \delta g_{L}^{t T}=\frac{s_{L} c_{L}}{2} \\
\delta g_{R}^{i}=\delta g_{R}^{i j}=0, \quad i, j=t, T .
\end{array}
$$

Finally, the unitarity bound from the scattering $T \bar{T} \rightarrow T \bar{T}$ is modified from the Standard Model limit and becomes [65, 66]

$$
M_{T}(\text { Unitarity Bound }) \lesssim \frac{550 \mathrm{GeV}}{s_{L}^{2}} .
$$

This class of models therefore allows quite heavy $T$ quarks without violating perturbative unitarity.

\section{A. Experimental Restrictions on Singlet Top Partner Model}

Using the results given above, it is straightforward to compute the contributions to the $S, T$ and $U$ parameters in the singlet top partner model. Expressions for the gauge boson 
two-point functions for arbitrary fermion couplings are given in the appendix [21, 67, 68], and we assume $M_{T}>>M_{Z}$. Subtracting the Standard Model $t-b$ loops, the new contributions are

$$
\begin{aligned}
\Delta T_{F}= & T_{S M} s_{L}^{2}\left[-\left(1+c_{L}^{2}\right)+s_{L}^{2} r+2 c_{L}^{2} \frac{r}{r-1} \log (r)+\mathcal{O}\left(\frac{M_{Z}^{2}}{m_{t}^{2}}, \frac{M_{Z}^{2}}{M_{T}^{2}}, \frac{m_{b}^{2}}{m_{t}^{2}}\right)\right] \\
\Delta S_{F}= & -\frac{N_{C}}{18 \pi} s_{L}^{2}\left\{\log (r)+c_{L}^{2}\left[\frac{5\left(r^{2}+1\right)-22 r}{(r-1)^{2}}+\frac{3(r+1)\left(r^{2}-4 r+1\right)}{(1-r)^{3}} \log (r)\right]\right. \\
& \left.+\mathcal{O}\left(\frac{M_{Z}^{2}}{m_{t}^{2}}, \frac{M_{Z}^{2}}{M_{T}^{2}}, \frac{m_{b}^{2}}{m_{t}^{2}}\right)\right\} \\
\Delta S_{F}+\Delta U_{F}= & \frac{N_{C}}{9 \pi} s_{L}^{2}\left[\log (r)+\mathcal{O}\left(\frac{M_{Z}^{2}}{m_{t}^{2}}, \frac{M_{Z}^{2}}{M_{T}^{2}}, \frac{m_{b}^{2}}{m_{t}^{2}}\right)\right]
\end{aligned}
$$

where

$$
r \equiv \frac{M_{T}^{2}}{m_{t}^{2}} \quad, \quad N_{C}=3 \quad, \quad \text { and } \quad T_{S M}=\frac{N_{C}}{16 \pi s_{W}^{2}} \frac{m_{t}^{2}}{M_{W}^{2}}
$$

is the $t-b$ contribution to the $T$ parameter in the limit of a massless bottom quark. We use $M_{W}=80.4 \mathrm{GeV}, M_{Z}=91.2 \mathrm{GeV}$ [37]. Eq. 30 agrees with the $m_{b} \rightarrow 0$ and $M_{Z}<<M_{T}, m_{t}$ limits of Refs. [69 71]. ${ }^{3}$ For a top parter much heavier than the top quark, $M_{T}>>m_{t}$, the oblique parameters take simple forms,

$$
\begin{aligned}
& \Delta T_{F} \text { (approx) }=T_{S M} s_{L}^{2}\left[-\left(1+c_{L}^{2}\right)+s_{L}^{2} r+2 c_{L}^{2} \log (r)\right] \\
& \Delta S_{F}(\text { approx })=-\frac{N_{C}}{18 \pi} s_{L}^{2}\left[5 c_{L}^{2}+\left(1-3 c_{L}^{2}\right) \log (r)\right] .
\end{aligned}
$$

One would expect decoupling to occur for a very heavy vector top partner, i.e. for $r \rightarrow \infty$. From Eqs. 30, 32, instead, the effects of the top partner on the oblique parameters vanish only in the limit $s_{L} \rightarrow 0$. This can be understood inspecting the mass matrix (18): to obtain decoupling both the Yukawa interactions and the off-diagonal terms need to be small, $\lambda_{2} v, \lambda_{3} v, \lambda_{4}<<\lambda_{5}$. In this limit

$$
s_{L} \rightarrow \frac{v \lambda_{3}}{\sqrt{2} M_{T}}+\frac{\lambda_{2} \lambda_{4} v}{\sqrt{2} M_{T}^{2}}+\ldots
$$

and the top partner effects vanish for large $M_{T}$, as expected.

In Fig. 2 we show the oblique parameters for a fixed $M_{T}=1 \mathrm{TeV}$. It is clear that the approximate forms of Eq. 32 are excellent approximations to the complete results for mass

3 There is a typo in Eq. 88 of Ref. [70], where there is a 2 instead of the factor 22 in Eq. 30 


\section{Singlet Fermion Model}

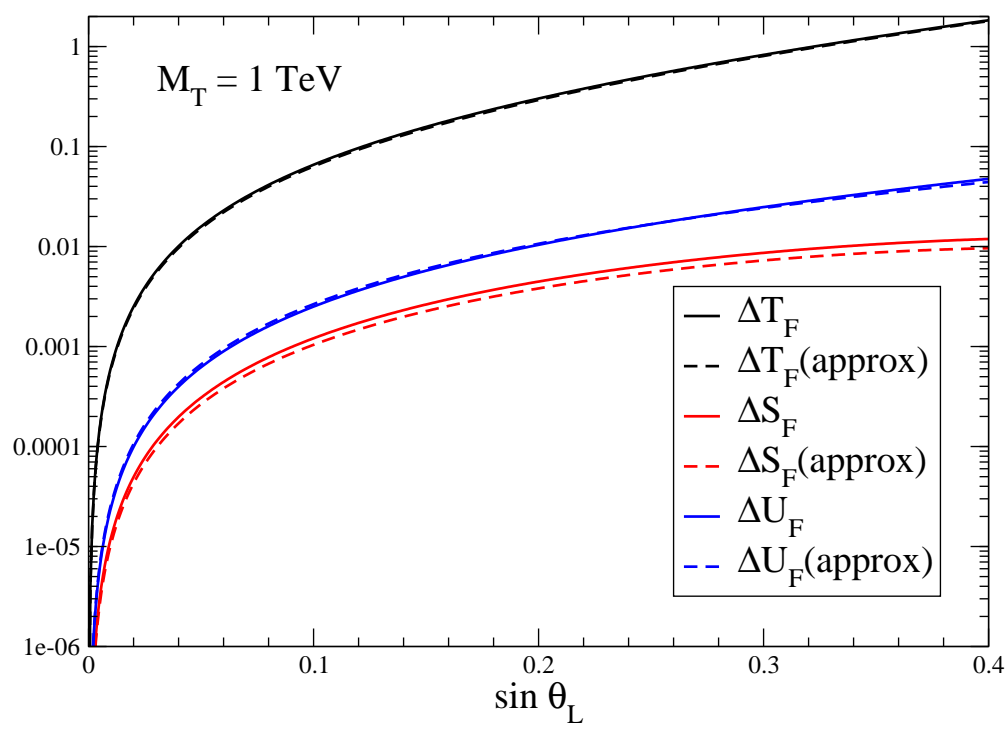

FIG. 2: Contributions to $\Delta T_{F}, \Delta S_{F}, \Delta U_{F}$ from a singlet top partner as a function of $\sin \theta_{L}$ for fixed $M_{T}=1 \mathrm{TeV}$. The results of Eq. 32 in the limit $M_{T}>>m_{t}$ are shown as $\Delta T_{F}$ (approx), $\Delta S_{F}($ approx $)$ and $\Delta U_{F}($ approx $)$.

\section{Singlet Fermion Model}

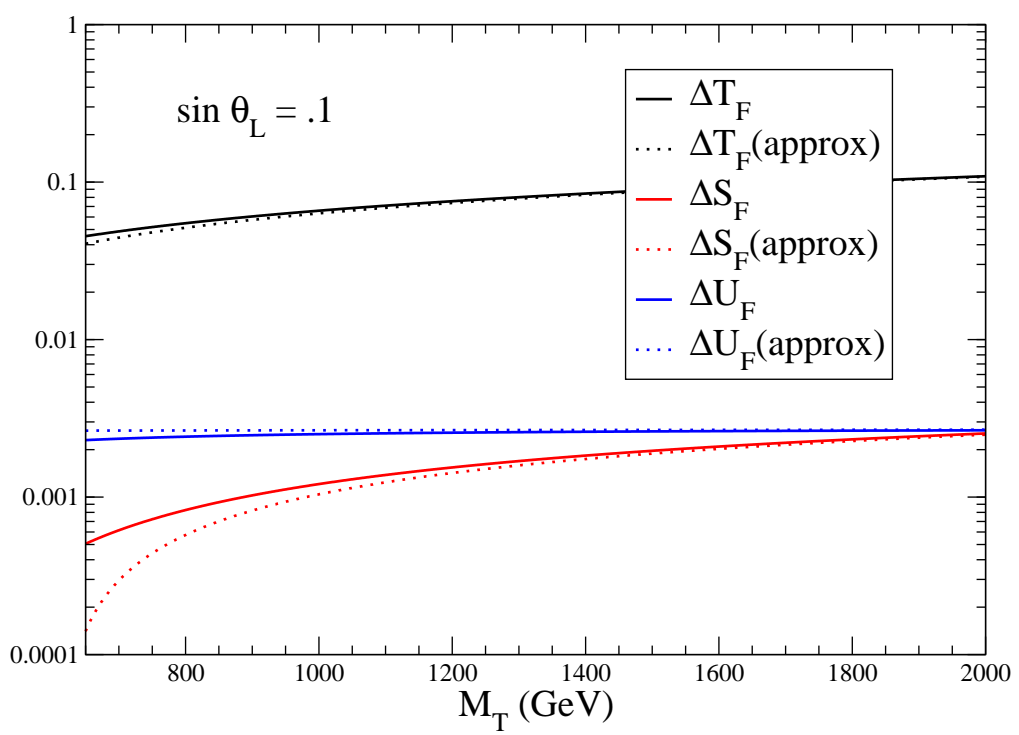

FIG. 3: Fermion contributions to $\Delta T_{F}, \Delta S_{F}, \Delta U_{F}$ in the singlet top partner model for fixed $\sin \theta_{L}=0.1$. The dotted lines represent the approximate results from Eq. 32 in the limit $M_{T}>m_{t}$. 


\section{Singlet Fermion Model}

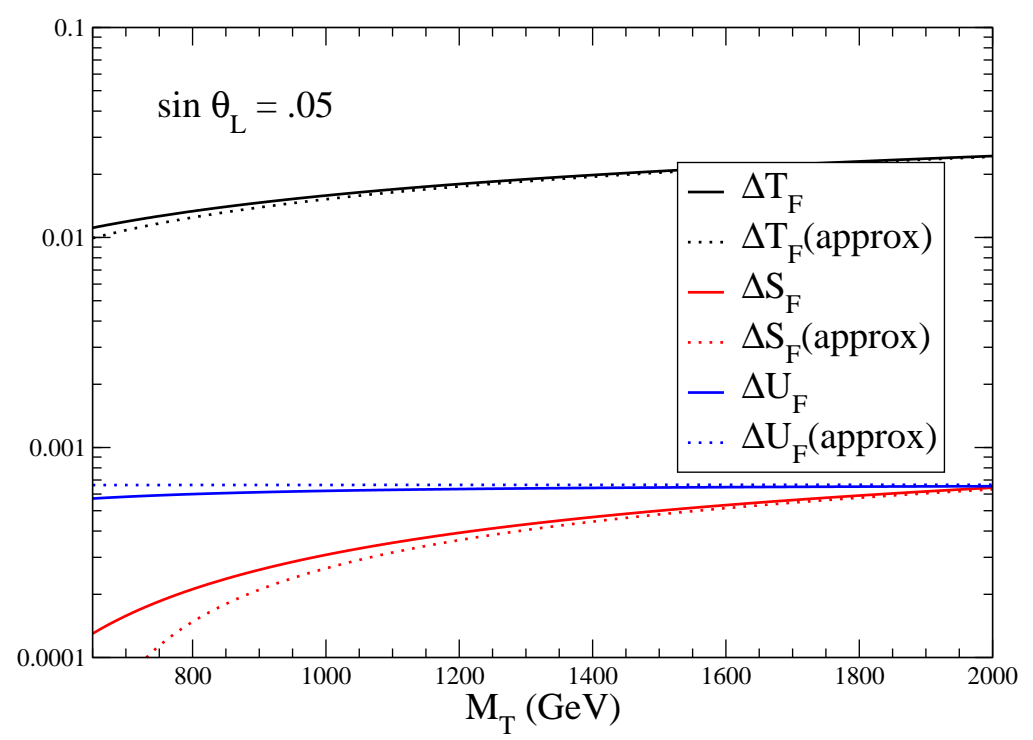

FIG. 4: Same as Fig. 3 for a smaller $\sin \theta_{L}=0.05$.

values of this order. The largest contribution is to $\Delta T_{F}$ due to the large isospin violation for non-zero $\sin \theta_{L}$. In this case, the isospin violation is manifest in the result that $\Delta U_{F}>\Delta S_{F}$. The new physics effects vanish as $s_{L} \rightarrow 0$ and we recover the Standard Model couplings. The oblique parameters for fixed $\sin \theta_{L}$ are shown in Figs. 3 and 4 as a function of $M_{T}$. As we argued, the decoupling does not occur for large $M_{T}$, but requires $s_{L} \rightarrow 0$.

The parameter space allowed by a fit to the oblique parameters can be found using the results of Section III. Fig. 5 shows the 95\% confidence level upper bound on the mixing angle $\sin \theta_{L}$ as a function of $M_{T}$ for a light Higgs boson. For a heavier Higgs boson, it is possible to use the positive contribution to $T$ from the top partner to compensate for the negative contribution from the heavy Higgs, as shown in Fig. 6. In this case, a minimum degree of mixing is required, since such a heavy Higgs boson is excluded by the electroweak fit in the three-generation Standard Model. The heavier the Higgs boson, the smaller the range of $\sin \theta_{L}$ allowed. This situation was explored for an extremely heavy Higgs boson $\left(M_{H} \sim 800 \mathrm{GeV}\right)$ in Ref. [72].

The mixing in the charge $2 / 3$ sector also affects $R_{b}$. In the limit $m_{b} \rightarrow 0$ and neglecting the small correlations between $R_{b}$ and the oblique parameters, only $\delta g_{L}^{b}$ is affected by the singlet 
95\% Confidence Level Upper Bound from STU

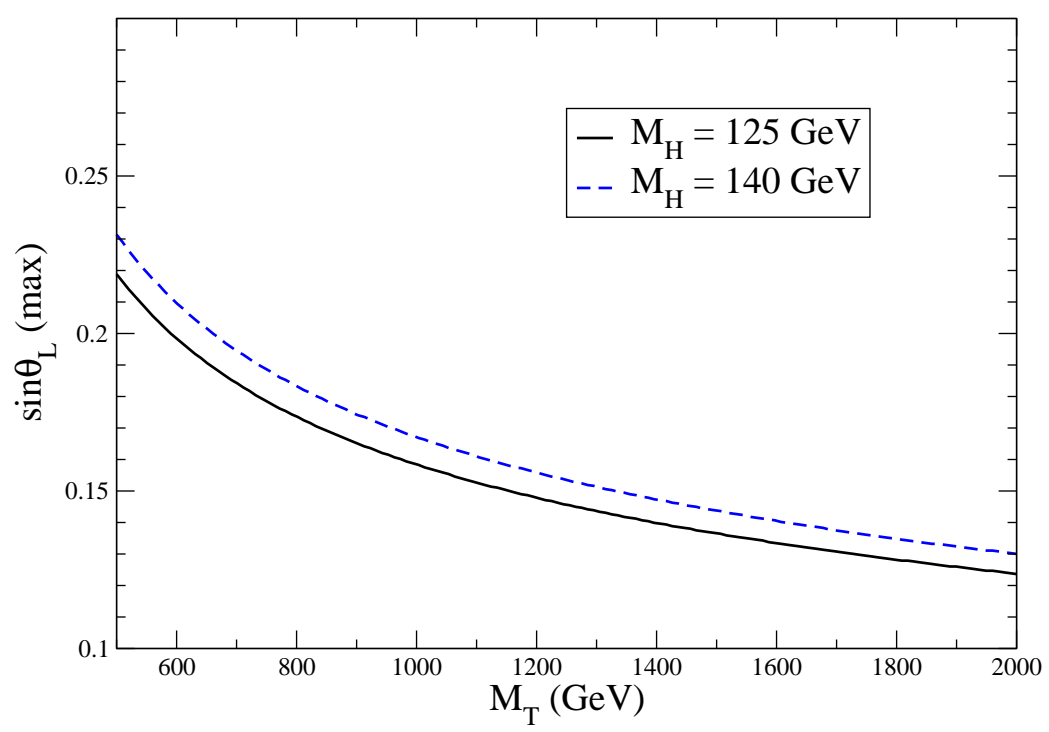

FIG. 5: $95 \%$ confidence level upper bound on the mixing angle $\sin \theta_{L}$ in the singlet top partner model from experimental restrictions on the $S, T$ and $U$ parameters.

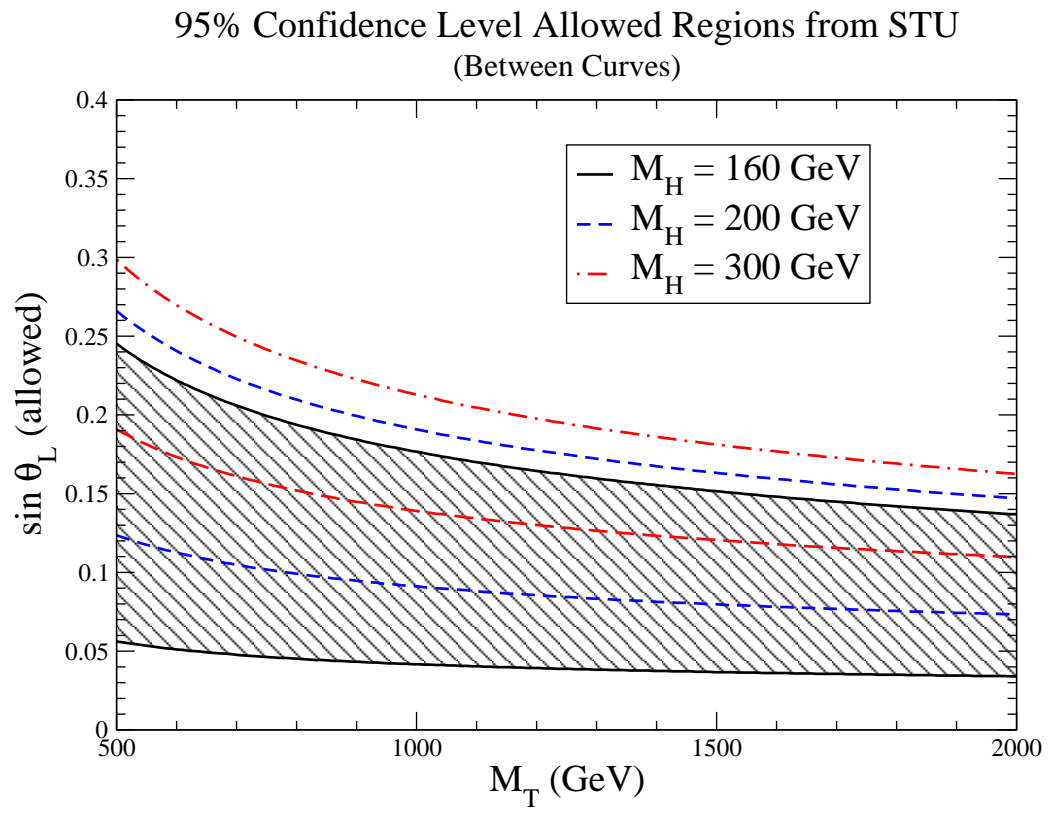

FIG. 6: $95 \%$ confidence level bands allowed by a fit to $S, T$ and $U$ for the mixing angle $\sin \theta_{L}$ in the singlet top partner model. The regions allowed are between the curves corresponding to each value of $M_{H}$. 
top partner. Its contribution to $\delta g_{L}^{b}$ can be found from the general analysis of Ref. [73],

$$
\delta g_{L}^{b}=\frac{g^{2}}{64 \pi^{2}} s_{L}^{2}\left(f_{1}\left(x, x^{\prime}\right)+c_{L}^{2} f_{2}\left(x, x^{\prime}\right)\right)
$$

where $x=m_{t}^{2} / M_{W}^{2}$ and $x^{\prime}=M_{T}^{2} / M_{W}^{2}$. The Standard Model top contribution has been subtracted following the definition of Eq. 5. In the heavy mass limit, $x, x^{\prime}>>1$,

$$
\begin{aligned}
& f_{1}\left(x, x^{\prime}\right)=x^{\prime}-x+3 \log \left(\frac{x^{\prime}}{x}\right) \\
& f_{2}\left(x, x^{\prime}\right)=-x-x^{\prime}+\frac{2 x x^{\prime}}{x^{\prime}-x} \log \left(\frac{x^{\prime}}{x}\right) .
\end{aligned}
$$

The contribution to $\delta g_{L}^{b}$ from top singlet partners is shown in Fig. 7 as a function of $M_{T}$ for fixed $\sin \theta_{L}$, along with the $95 \%$ confidence level region allowed from the fit of Section IIA. We use the exact one-loop calculation of $\delta g_{L}^{b}$ in the top singlet partner model, following Refs. [73, 74]. The relatively large contributions from $R_{b}$ can be understood by looking at the leading terms for $m_{t}, M_{T}>>M_{W}$,

$$
\delta g_{L}^{b}=\delta g_{L}^{b, S M} s_{L}^{2}\left[-\left(1+c_{L}^{2}\right)+s_{L}^{2} r+2 c_{L}^{2} \frac{r}{r-1} \log (r)\right] .
$$

Again we see that the heavy top partner decouples only when the parameters in the mass matrix are such that $s_{L} \sim \frac{v}{M_{T}}$. Furthermore, its contributions to $\delta g_{L}^{b}$ and to the $T$ parameter (Eq. 32) are both positive and strongly correlated. A large, positive contribution to $\delta g_{L}^{b}$ from the singlet also implies a large contribution to the $T$ parameter. This correlation was already pointed out in the context of composite Higgs models in Refs. [31, 32, 75].

Combining the new physics contribution with the Standard Model top quark contribution,

$$
\delta \tilde{g}_{L}^{b}=\delta g_{L}^{b, S M}+\delta g_{L}^{b}=\delta g_{L}^{b, S M}\left[c_{L}^{4}+s_{L}^{4} r+2 s_{L}^{2} c_{L}^{2} \frac{r}{r-1} \log (r)\right],
$$

and the net effect of the top partner is to increase $\delta \tilde{g}_{L}^{b}$ and hence reduce $R_{b}$.

A comparison of the maximum value of $\sin \theta_{L}$ allowed by the fit to $R_{b}$ and $A_{b}$ and by the experimental limits arising from the fit to $S, T$ and $U$ (with $M_{H}=125 \mathrm{GeV}$ ) is shown in Fig. 8, where it is apparent that the most stringent restrictions in the top partner singlet model come from the oblique parameters. 
Singlet Fermion Model

95\% Confidence Level Limit from $\mathrm{Z} \rightarrow \mathrm{bb}$ and $\mathrm{A}_{\mathrm{b}}$

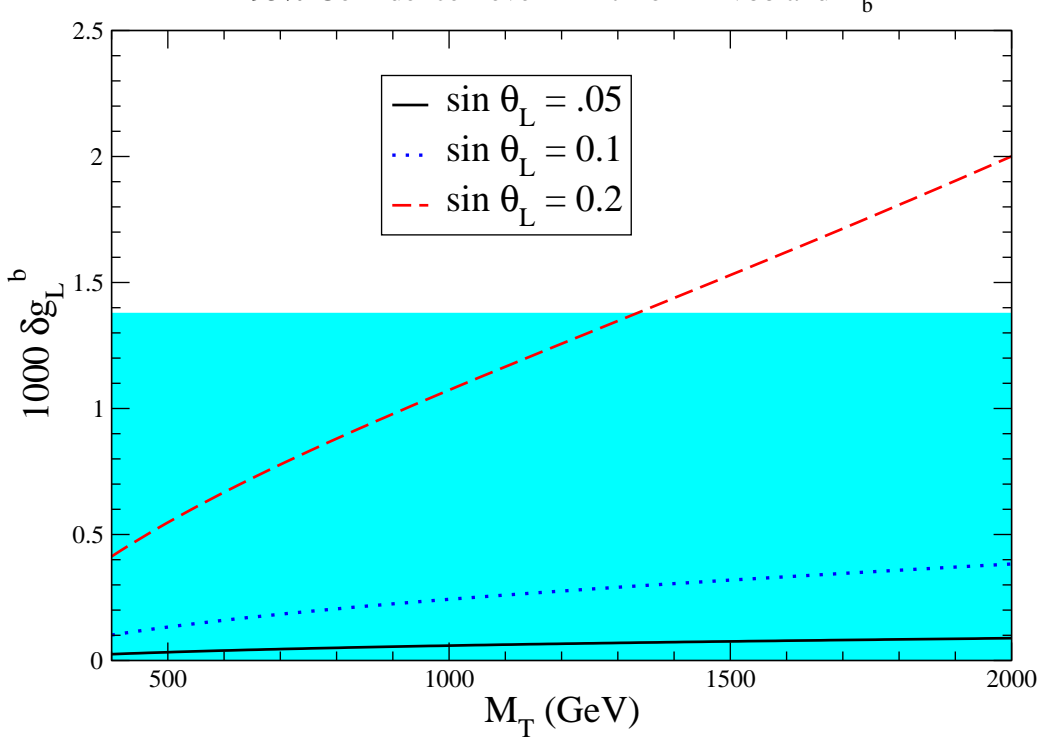

FIG. 7: Fermion contributions to $\delta g_{L}^{b}$ as a function of $M_{T}$ for fixed $\sin \theta_{L}$ in the top partner singlet model. The $95 \%$ confidence level allowed region is shaded in light blue.

95\% Confidence Level Upper Limits

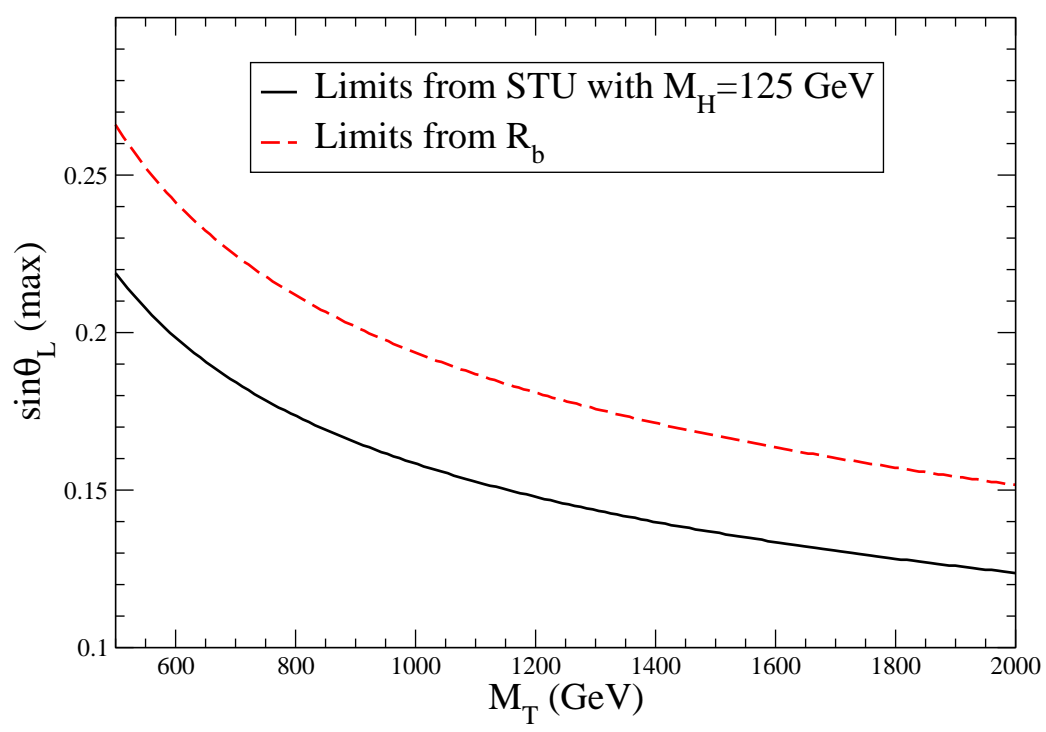

FIG. 8: Maximum allowed $\sin \theta_{L}$ in the top partner singlet model from oblique measurements (black solid) and $R_{b}$ (red dashed). 


\section{TOP PARTNER DOUBLET}

\section{A. Model with Top Partner Doublet}

In this section, we consider a model which has in addition to the Standard Model fields a vector $S U(2)_{L}$ doublet [36, 76],

$$
\psi_{L}^{2}=\left(\begin{array}{c}
\mathcal{T}_{L}^{2} \\
\mathcal{B}_{L}^{2}
\end{array}\right), \quad \psi_{R}^{2}=\left(\begin{array}{c}
\mathcal{T}_{R}^{2} \\
\mathcal{B}_{R}^{2}
\end{array}\right) .
$$

The most general fermion mass terms allowed in the Lagrangian are

$$
\begin{aligned}
-\mathcal{L}_{M, 2} & =-\mathcal{L}_{M}^{S M}+\lambda_{6} \bar{\psi}_{L}^{2} H \mathcal{B}_{R}^{1}+\lambda_{7} \bar{\psi}_{L}^{2} \tilde{H} \mathcal{T}_{R}^{1}+\lambda_{8} \bar{\psi}_{L}^{2} \psi_{R}^{2}+\lambda_{9} \bar{\psi}_{L}^{1} \psi_{R}^{2}+\text { h.c. } \\
& =\bar{\chi}_{L}^{t}\left[U_{L}^{t} M_{(2)}^{t} U_{R}^{t \dagger}\right] \chi_{R}^{t}+\bar{\chi}_{L}^{b}\left[U_{L}^{b} M_{(2)}^{b} U_{R}^{b \dagger}\right] \chi_{R}^{b}+\text { h.c. },
\end{aligned}
$$

where $\chi_{L, R}^{t}$ are given by Eq. [15 and

$$
\chi_{L, R}^{b} \equiv\left(\begin{array}{c}
b_{L, R} \\
B_{L, R}
\end{array}\right) \equiv U_{L, R}^{b}\left(\begin{array}{c}
\mathcal{B}_{L, R}^{1} \\
\mathcal{B}_{L, R}^{2}
\end{array}\right) .
$$

We can always rotate $\psi_{2}$ such that $\lambda_{9}=0$. So without loss of generality

$$
M_{(2)}^{t}=\left(\begin{array}{cc}
\lambda_{2} \frac{v}{\sqrt{2}} & 0 \\
\lambda_{7} \frac{v}{\sqrt{2}} & \lambda_{8}
\end{array}\right), \quad M_{(2)}^{b}=\left(\begin{array}{cc}
\lambda_{1} \frac{v}{\sqrt{2}} & 0 \\
\lambda_{6} \frac{v}{\sqrt{2}} & \lambda_{8}
\end{array}\right) .
$$

Because of the $S U(2)$ symmetry

$$
M_{(2), 22}^{t}=M_{(2), 22}^{b} .
$$

Diagonalizing the mass matrices now requires four unitary mixing matrices, $U_{L}^{t}, U_{R}^{t}, U_{L}^{b}, U_{R}^{b}$,

$$
\begin{aligned}
& U_{L}^{t}=\left(\begin{array}{cc}
\cos \theta_{L}^{t} & -\sin \theta_{L}^{t} \\
\sin \theta_{L}^{t} & \cos \theta_{L}^{t}
\end{array}\right), \quad U_{R}^{t}=\left(\begin{array}{cc}
\cos \theta_{R}^{t} & -\sin \theta_{R}^{t} \\
\sin \theta_{R}^{t} & \cos \theta_{R}^{t}
\end{array}\right), \\
& U_{L}^{b}=\left(\begin{array}{cc}
\cos \theta_{L}^{b} & -\sin \theta_{L}^{b} \\
\sin \theta_{L}^{b} & \cos \theta_{L}^{b}
\end{array}\right), \quad U_{R}^{b}=\left(\begin{array}{cc}
\cos \theta_{R}^{b} & -\sin \theta_{R}^{b} \\
\sin \theta_{R}^{b} & \cos \theta_{R}^{b}
\end{array}\right) .
\end{aligned}
$$

There are five input parameters in the Lagrangian. We will take the five independent physical parameters to be the physical masses, $m_{t}, M_{T}$ and $m_{b}, M_{B}$ (with $m_{t}$ and $m_{b}$ being 
the mass of the Standard Model-like fermions) and the right-handed mixing angle in the charge $-1 / 3$ sector, $\theta_{R}^{b}$. It is straightforward to find relationships among the mixing angles:

$$
\begin{aligned}
\left(\sin \theta_{R}^{t}\right)^{2} & =\frac{\left(\sin \theta_{L}^{t}\right)^{2}}{\left(\sin \theta_{L}^{t}\right)^{2}+\left(\cos \theta_{L}^{t}\right)^{2} \frac{m_{t}^{2}}{M_{T}^{2}}} \\
\left(\sin \theta_{R}^{b}\right)^{2} & =\frac{\left(\sin \theta_{L}^{b}\right)^{2}}{\left(\sin \theta_{L}^{b}\right)^{2}+\left(\cos \theta_{L}^{b}\right)^{2} \frac{m_{b}^{2}}{M_{B}^{2}}} \\
\left(\sin \theta_{R}^{b}\right)^{2}\left(m_{b}^{2}-M_{B}^{2}\right)+M_{B}^{2} & =\left(\sin \theta_{R}^{t}\right)^{2}\left(m_{t}^{2}-M_{T}^{2}\right)+M_{T}^{2} .
\end{aligned}
$$

For small mixing, the left-handed angles are always suppressed by the heavy mass scale relative to the right-handed angles of the same charge sector,

$$
\sin ^{2} \theta_{L}^{t, b} \sim \frac{m_{t, b}^{2}}{M_{T, B}^{2}} \sin ^{2} \theta_{R}^{t, b} .
$$

If the mass splitting between $B$ and $T, \delta \equiv M_{T}-M_{B}$, is small, $\frac{|\delta|}{M_{T}}<<1$,

$$
\left(\sin \theta_{R}^{t}\right)^{2}=\left(\sin \theta_{R}^{b}\right)^{2}+\left(\cos \theta_{R}^{b}\right)^{2} \frac{2 \delta}{M_{T}}+\mathcal{O}\left(\frac{\delta^{2}}{M_{T}^{2}}, \frac{m_{t}^{2}}{M_{T}^{2}}, \frac{m_{b}^{2}}{M_{T}^{2}}\right) .
$$

The charged current interactions are

$$
\begin{aligned}
\mathcal{L}_{2}^{C C} & =\frac{g}{\sqrt{2}} W^{\mu+}\left\{\left[\Sigma_{i=1}^{2} \bar{\psi}_{L}^{i} \gamma_{\mu} \sigma^{-} \psi_{L}^{i}\right]+\bar{\psi}_{R}^{2} \gamma_{\mu} \sigma^{-} \psi_{R}^{2}\right\}+\text { h.c. } \\
& =\frac{g}{\sqrt{2}} W^{\mu+}\left\{\bar{\chi}_{L}^{t} \gamma_{\mu} U_{L}^{t} U_{L}^{b, \dagger} \chi_{L}^{b}+\bar{\chi}_{R}^{t} \gamma_{\mu} U_{R}^{t} U_{R}^{b, \dagger} \chi_{R}^{b}\right\}+\text { h.c. }
\end{aligned}
$$

where

$$
\sigma^{-}=\left(\begin{array}{ll}
0 & 1 \\
0 & 0
\end{array}\right)
$$

The neutral current interactions are given by Eq. 27, with

$$
\begin{aligned}
\delta g_{L}^{i}=\delta g_{L}^{i j}=0, & i, j=t, T, b, B & \\
\delta g_{R}^{t}=T_{3}^{t} \sin ^{2} \theta_{R}^{t}, & \delta g_{R}^{T}=T_{3}^{T} \cos ^{2} \theta_{R}^{t}, & \delta g_{R}^{t T}=-\frac{1}{2} \sin \theta_{R}^{t} \cos \theta_{R}^{t} \\
\delta g_{R}^{b}=T_{3}^{b} \sin ^{2} \theta_{R}^{b}, & \delta g_{R}^{B}=T_{3}^{b} \cos ^{2} \theta_{R}^{b}, & \delta g_{R}^{b B}=\frac{1}{2} \sin \theta_{R}^{b} \cos \theta_{R}^{b} .
\end{aligned}
$$

In the doublet top-partner model, only the right-handed Standard Model-like singlets $\mathcal{T}_{1}^{R}$, $\mathcal{B}_{1}^{R}$ have Yukawa type mixing with the new quarks, and therefore only the interactions in the right-handed sector are modified. Finally, the Higgs couplings are given by

$$
\begin{aligned}
\mathcal{L}_{2}^{h}= & -c_{t t} \frac{m_{t}}{v} \bar{t}_{L} t_{R} h-c_{T T} \frac{M_{T}}{v} \bar{T}_{L} T_{R} h-c_{t T} \frac{m_{t}}{v} \bar{t}_{L} T_{R} h-c_{T t} \frac{M_{T}}{v} \bar{T}_{L} t_{R} h+ \\
& -c_{b b} \frac{m_{b}}{v} \bar{b}_{L} b_{R} h-c_{B B} \frac{M_{B}}{v} \bar{B}_{L} B_{R} h-c_{b B} \frac{m_{b}}{v} \bar{b}_{L} B_{R} h-c_{B b} \frac{M_{B}}{v} \bar{B}_{L} b_{R} h+\text { h.c. },
\end{aligned}
$$


where

$$
\begin{array}{lll}
c_{t t}=\cos ^{2} \theta_{R}^{t}, & c_{T T}=\sin ^{2} \theta_{R}^{t}, & c_{t T}=c_{T t}=\sin \theta_{R}^{t} \cos \theta_{R}^{t} \\
c_{b b}=\cos ^{2} \theta_{R}^{b}, & c_{B B}=\sin ^{2} \theta_{R}^{b}, & c_{b B}=c_{B b}=\sin \theta_{R}^{b} \cos \theta_{R}^{b} .
\end{array}
$$

The derivation of these couplings follows the lines of Eq. 25, just with

$$
v H_{(2), k s}^{t}=M_{(1), k s}^{t} \delta_{s 1}
$$

\section{B. Experimental Limits on Doublet Fermion Model}

The decay $Z \rightarrow b \bar{b}$ puts a strong restriction on $\sin \theta_{b}^{R}$ since mixing in the right-handed $b$-quark sector contributes to $\delta g_{R}^{b}$ at tree level,

$$
\delta g_{R}^{b}=-\frac{1}{2}\left(\sin \theta_{R}^{b}\right)^{2}
$$

From Eq. 10, the mixing angle in the right-handed $b$ sector is highly constrained,

$$
\left|\sin \theta_{R}^{b}\right|<0.115
$$

The contribution to $\delta g_{L}^{b}$ in the left-handed sector occurs at one-loop. Subtracting out the Standard Model contribution, in the limit $x, x^{\prime}>>1$, the approximate result in the doublet fermion model is [34]

$$
\delta g_{L}^{b}=\frac{g^{2}}{64 \pi^{2}}\left\{\sin ^{2}\left(\theta_{L}^{t}-\theta_{L}^{b}\right) f_{1}\left(x, x^{\prime}\right)+\left(\sin \theta_{R}^{t}\right)^{2} \cos ^{2}\left(\theta_{L}^{t}-\theta_{L}^{b}\right) f_{3}\left(x, x^{\prime}\right)\right\},
$$

where $f_{1}\left(x, x^{\prime}\right)$ is defined in Eq. 35 and

$$
f_{3}\left(x, x^{\prime}\right)=-x+\frac{3}{2}\left(1+\frac{x}{x^{\prime}}\right)+\left(\frac{x^{\prime}+x}{2}-3\right) \frac{x}{\left(x^{\prime}-x\right)} \log \left(\frac{x^{\prime}}{x}\right) .
$$

In Fig. 9, we scan over $\sin \theta_{R}^{b}$ and $\frac{\delta}{M_{T}}$ and use the relationships of Eq.44to find the remaining parameters. We use the exact one-loop result for $\delta g_{b}^{L}$ following Refs. [34, 74], and determine the $95 \%$ confidence level upper bound on $\frac{\delta}{M_{T}}$. Because of the tree level mixing in the $b$ sector in this model, along with the relationships of Eq. 44, the heavy fermions are required to be approximately degenerate, as is clear from Fig. 9. This result is relatively insensitive to $M_{T}$.

Since $\sin \theta_{R}^{b}$ is constrained to be quite small, we will consider the oblique parameters in the limit $\theta_{R}^{b}=0$. From Eq. 44, $\theta_{R}^{b}=0$ implies $\theta_{L}^{b}=0$ and the free parameters are $m_{t}, m_{b}$, 
95\% Confidence Level Limits from $\mathrm{Z} \rightarrow \mathrm{bb}$ and $\mathrm{A}_{\mathrm{b}}$ Doublet Fermion Model

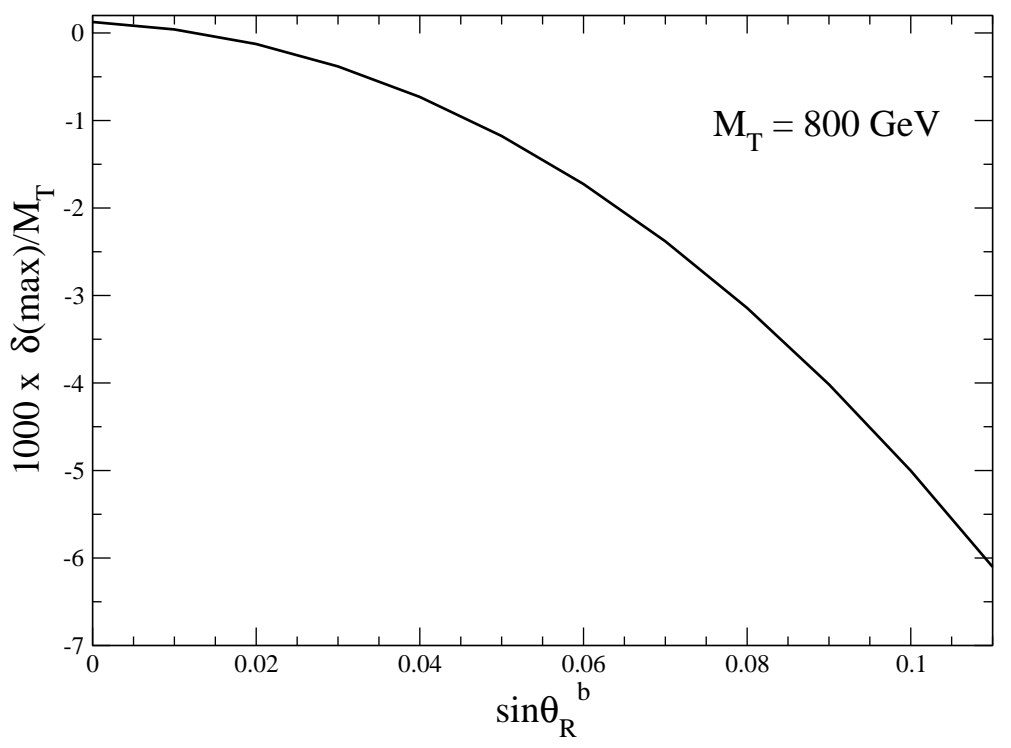

FIG. 9: Maximum value of $\frac{\delta}{M_{T}}$ allowed at the $95 \%$ confidence level from $R_{b}$ and $A_{b}$ as a function of $\sin \theta_{R}^{b}$ in the doublet fermion model.

\section{Doublet Fermion Model}

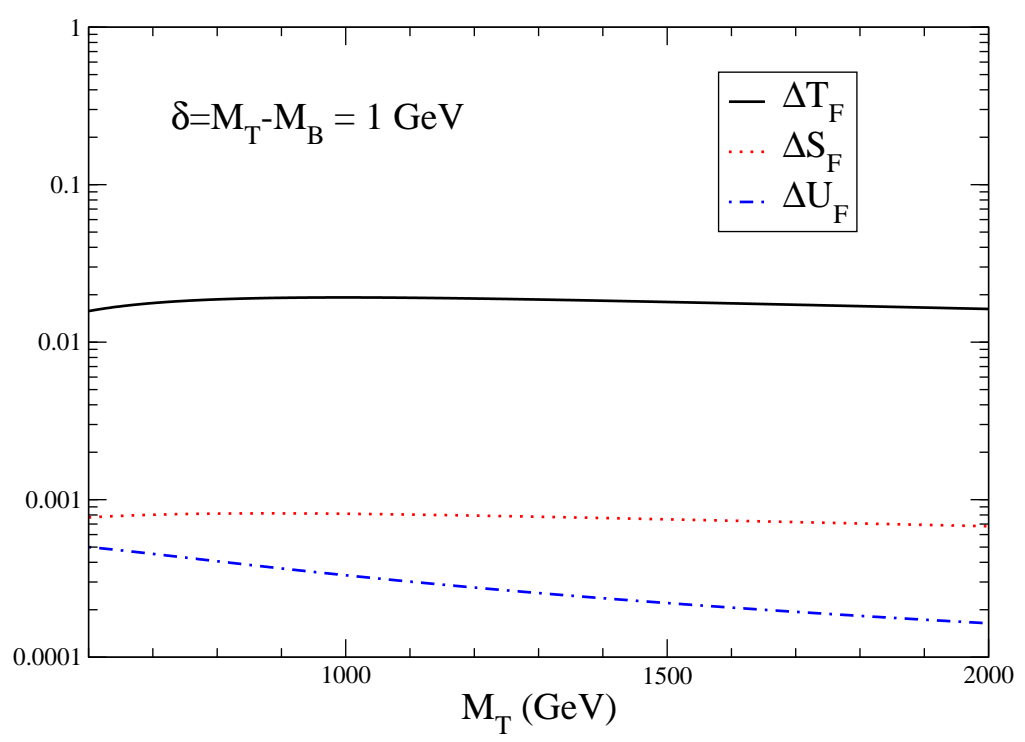

FIG. 10: Oblique parameters in the doublet fermion model in the limit $\theta_{R}^{b}=0$ and $m_{b} \rightarrow 0$ as a function of $M_{T}$. We fix $\delta=M_{T}-M_{B}=1 \mathrm{GeV}$. 
95\% Confidence Level Upper Bound from STU

Doublet Fermion Model

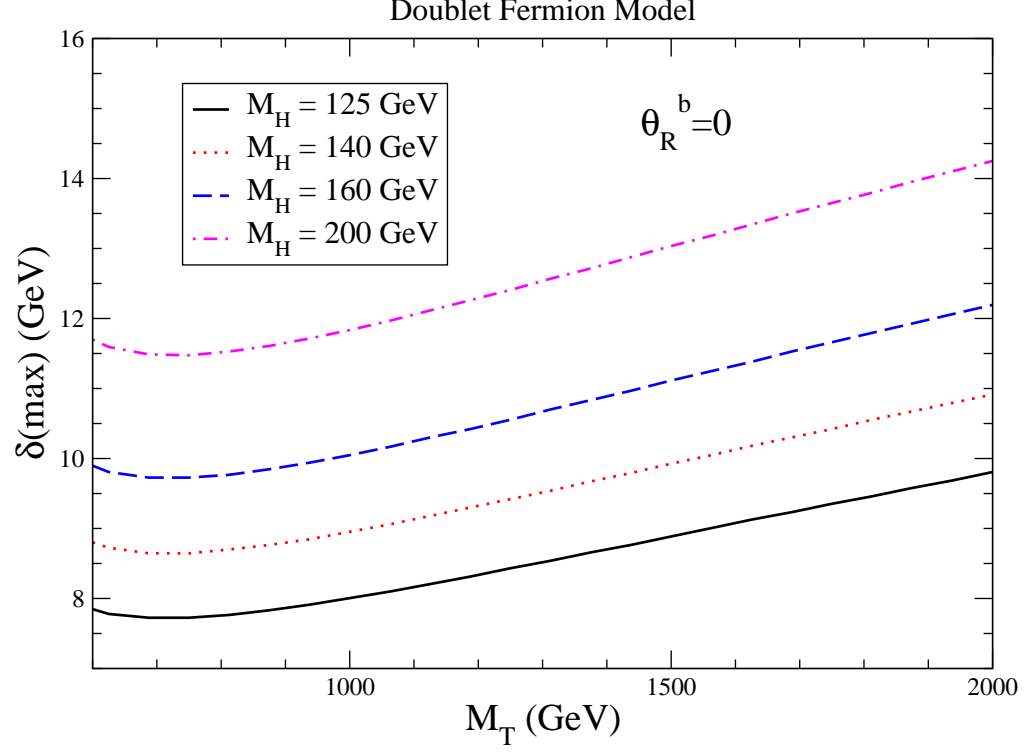

FIG. 11: Maximum mass splitting $\delta=M_{T}-M_{B}$ as a function of $M_{T}$ allowed at the $95 \%$ confidence level from the oblique parameters in the doublet fermion model for $\theta_{R}^{b}=0$.

$M_{T}$ and $M_{B}$. Our results will always be expressed in terms of $\delta \equiv M_{T}-M_{B}$. In the $\theta_{R}^{b}=0, m_{b} \rightarrow 0$ limit, the oblique parameters are well approximated by [69, 76]

$$
\begin{aligned}
\Delta T_{F}(\text { approx }) & =4 T_{S M} \frac{\delta}{M_{T}}\left[2 \log (r)-3+\frac{5 \log (r)-3}{r}\right] \\
\Delta S_{F}(\text { approx }) & =\frac{N_{C}}{9 \pi} \frac{\delta}{M_{T}}\left[4 \log (r)-7+\frac{4 \log (r)+7}{r}\right] \\
\Delta U_{F}(\text { approx }) & =\frac{N_{C}}{9 \pi} \frac{\delta}{M_{T}}\left[3+\frac{6 \log (r)-17}{r}\right],
\end{aligned}
$$

and

$$
\delta g_{b}^{L}=\delta g_{b}^{L, S M} \frac{\delta}{M_{T}}\left[\log (r)-4+3 \frac{\log (r)-2}{r}\right] .
$$

It is apparent that decoupling of the effects of the new fermions occurs in the isospin conserving limit, $\frac{\delta}{M_{T}} \rightarrow 0$. Note also that $\delta g_{b}^{L}$ changes sign for $M_{T} \simeq 7 m_{t}$. The oblique parameters are shown in Fig. 10 for $\delta=1 \mathrm{GeV}$. As in the singlet model, $\Delta T_{F}>>\Delta S_{F}, \Delta U_{F}$.

The limits coming from the oblique parameters are found from a global fit to $S, T$ and $U$ as described in Section ஹIB. For $\theta_{R}^{b}=0$, the $95 \%$ upper limit on the mass splitting, $\delta$, is shown in Fig. 11 as a function of $M_{T}$. For $M_{H}=125 \mathrm{GeV}$ and $M_{T} \sim 1 \mathrm{TeV}$, the experimental constraints on the oblique parameters require $\delta \lesssim 8 \mathrm{GeV}$. As shown in Fig. 11, it is possible 
to compensate for the negative contribution to $T$ from a heavier Higgs boson by a larger mass splitting $\delta$, which generates a positive contribution to $\Delta T_{F}$. However, the limits from $A_{b}$ and $R_{b}$ in this model are much more stringent than those coming from the oblique parameters.

\section{PHENOMENOLOGY}

The new fermions affect the gluon fusion production rate, which at lowest order is given by [77-79]

$$
\sigma(g g \rightarrow H)=\frac{\alpha_{s}^{2}}{1024 \pi v^{2}}\left|\Sigma_{q} c_{q q} F_{1 / 2}\left(\tau_{q}\right)\right|^{2} \delta\left(1-\frac{\hat{s}}{M_{H}^{2}}\right) .
$$

The sum is over $t, b, T$ in the singlet fermion model and over $t, b, T, B$ in the doublet model, the Yukawa couplings normalized to the Standard Model values $c_{q q}$ are given in Eqs. 23 and 51, and

$$
\begin{aligned}
\tau_{q} & =\frac{4 M_{q}^{2}}{M_{H}^{2}} \\
F_{1 / 2} & =-2 \tau_{q}\left[1+\left(1-\tau_{q}\right) f\left(\tau_{q}\right)\right] \\
f\left(\tau_{q}\right) & =\left\{\begin{array}{cc}
{\left[\sin ^{-1}\left(\sqrt{\frac{1}{\tau_{q}}}\right)\right]^{2},} & \text { if } \tau_{q} \geq 1 \\
-\frac{1}{4}\left[\log \left(\frac{1+\sqrt{1-\tau_{q}}}{1-\sqrt{1-\tau_{q}}}-i \pi\right]^{2},\right. & \text { if } \tau_{q}<1 .
\end{array}\right.
\end{aligned}
$$

We compute the gluon fusion production cross section through NNLO using the program iHixs [8]. iHixs allows the calculation of the cross section at NNLO for extensions of the Standard Model with an arbitrary number of heavy quarks having non-standard Yukawa interactions [13], and puts the predictions on a firm theoretical basis. At NNLO, there are contributions which mix quark loops of different flavors (e.g. $t$ and $T$ ) and cannot be obtained by a simple rescaling of the lowest order cross section. We scan the parameter

space allowed by the precision electroweak data as determined in the previous sections and discuss the maximum deviations from the Standard Model predictions.

\section{A. Top Partner Singlet Model}

The deviation from the Standard Model prediction of the NNLO Higgs production cross section as a function of the mixing angle in the top partner singlet model is shown in 


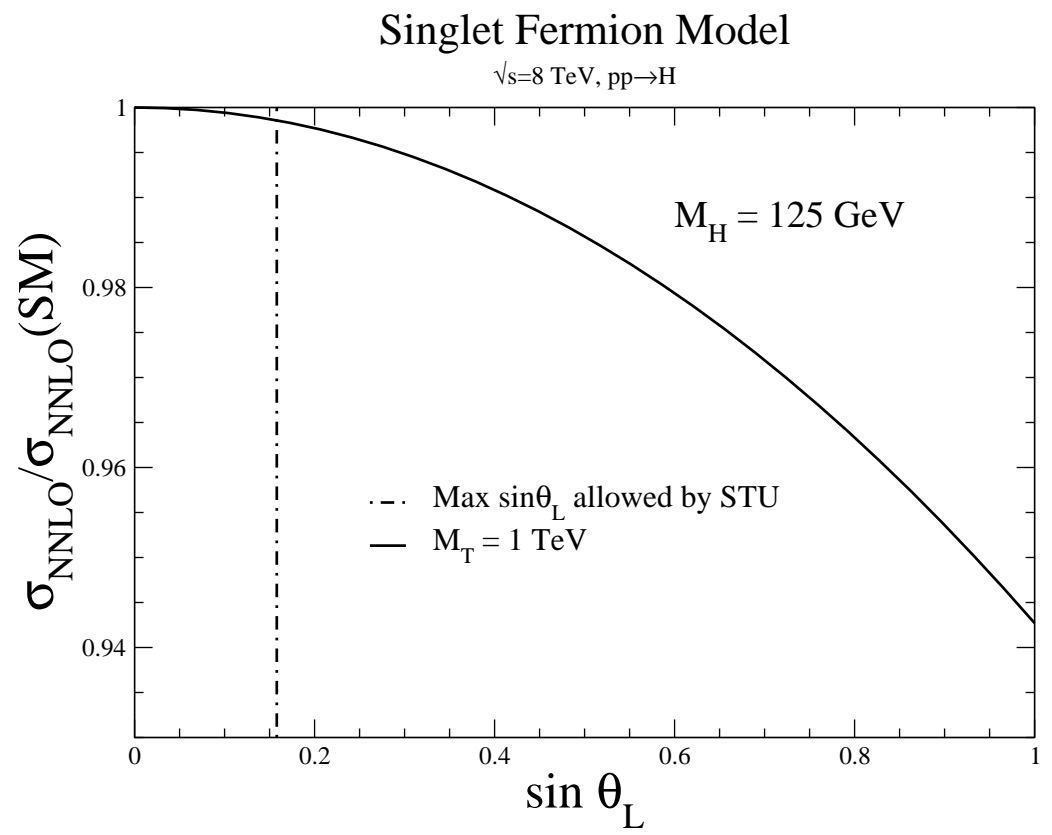

FIG. 12: The ratio of the NNLO Higgs cross section in the top partner singlet model normalized to the Standard Model prediction as a function of $\sin \theta_{L}$ for $M_{H}=125 \mathrm{GeV}$ and $\sqrt{s}=8 \mathrm{TeV}$. The vertical line represents the maximum value of $\sin \theta_{L}$ allowed by electroweak precision measurements.

Fig. 12. The largest value of $\sin \theta_{L}$ allowed by the precision electroweak limits derived in Section IVA is also shown. As $\sin \theta_{L}$ increases, the mixing with the Standard Model-like top quark becomes significant, causing a suppression of the rate. This can be understood from the heavy mass limit $\left(m_{t}, M_{T}>>\frac{M_{H}}{2}\right)$ of the lowest order cross section, where the gluon fusion rate scales as

$$
\frac{\sigma_{\text {Singlet }}}{\sigma_{S M}} \sim 1-\frac{7}{60} \frac{M_{H}^{2}}{m_{t}^{2}} s_{L}^{2}\left(1-\frac{m_{t}^{2}}{M_{T}^{2}}\right) .
$$

However, only the region to the left of the dot-dash line in Fig. 12] is allowed by the precision electroweak measurements, making the Higgs boson production rate in this model almost identical to the Standard Model rate. In contrast with composite [80] or Little Higgs [59, 81] models, which typically have a sizeable reduction of the Higgs production cross section relative to the Standard Model, in models with vector fermions the suppression is negligible because of the decoupling properties discussed in the previous sections. The uncertainty on the Standard Model cross section coming from scale, PDF, and $\alpha_{s}$ uncertainties is roughly $15-20 \%$ [7], so the extremely small deviation from the Standard Model prediction in the top partner singlet model is unobservable. The cross section for a heavier Higgs boson of 


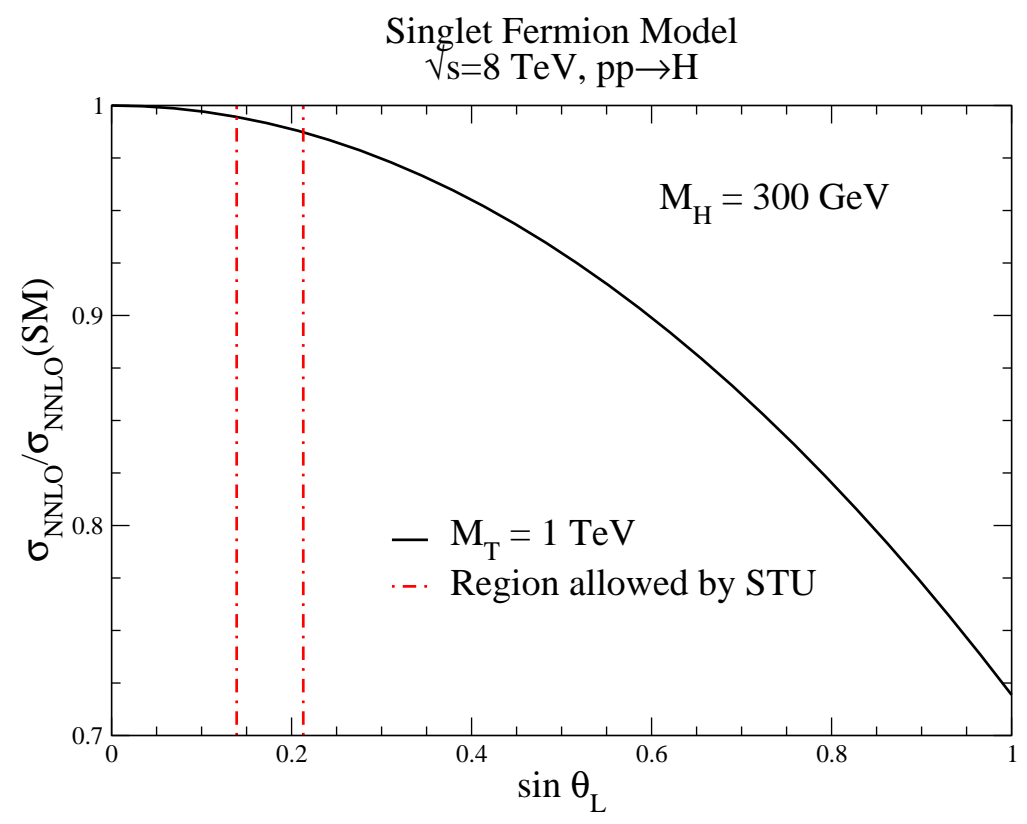

FIG. 13: The ratio of the NNLO Higgs cross section in the top partner singlet model normalized to the Standard Model prediction as a function of $\sin \theta_{L}$ for $M_{H}=300 \mathrm{GeV}$ and $\sqrt{s}=8 \mathrm{TeV}$. The region between the vertical lines represents the region of $\sin \theta_{L}$ allowed by electroweak precision measurements.

Singlet Fermion Model

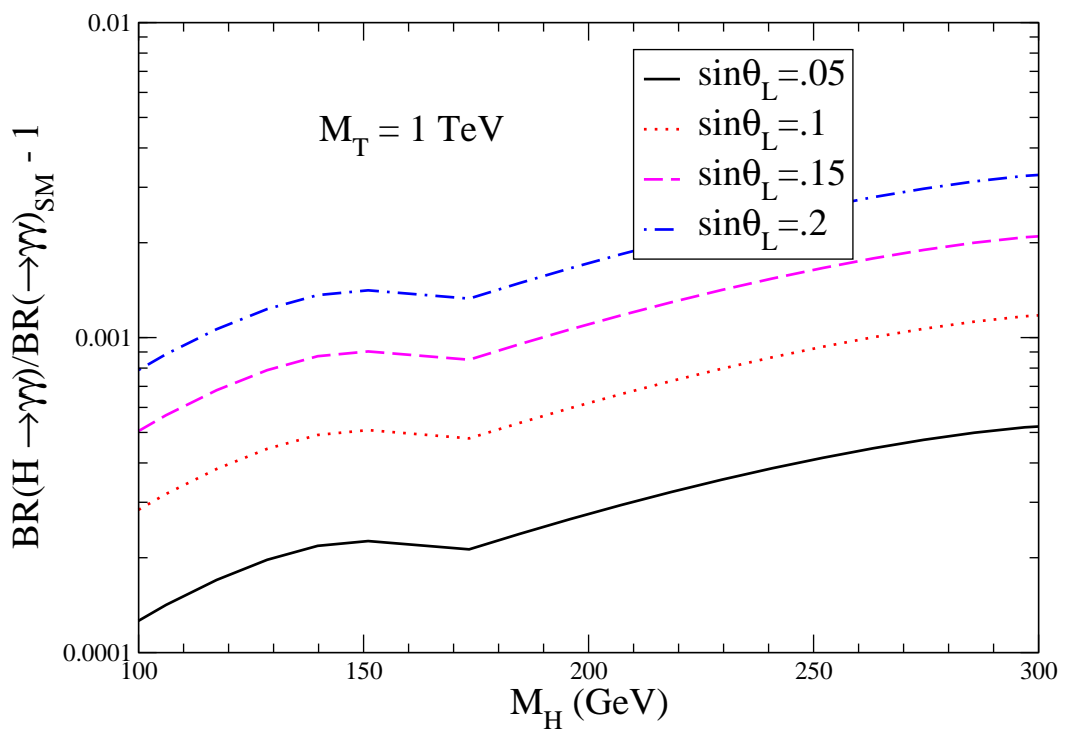

FIG. 14: Deviation of the $H \rightarrow \gamma \gamma$ branching ratio in the top partner singlet model from the Standard Model prediction. 
mass $M_{H}=300 \mathrm{GeV}$ is shown in Fig. 13, In this case, there is a region of mixing angles, $\sin \theta_{L}$, which is allowed by the precision electroweak measurements. Again, there is a slight, but unobservable, suppression of the NNLO rate relative to the Standard Model rate.

The loop-mediated Higgs decays to $\gamma \gamma, Z \gamma$ and $g g$ are also affected by the presence of top fermion partners. Fig. 14 shows the deviation of the branching ratio to $\gamma \gamma$ from the Standard Model prediction. For small mixing, this deviation is always less than one percent.

\section{B. Top Partner Doublet Model}

The deviation from the Standard Model prediction for the NNLO gluon fusion cross section for Higgs production in the top partner doublet model (computed using iHixs) is shown in Fig. 15. Also in this case the maximum difference from the Standard Model in the allowed regions of parameter space (Fig. 9) is always less than a few percent. This result can be understood by considering the heavy mass limit of the lowest order cross section for

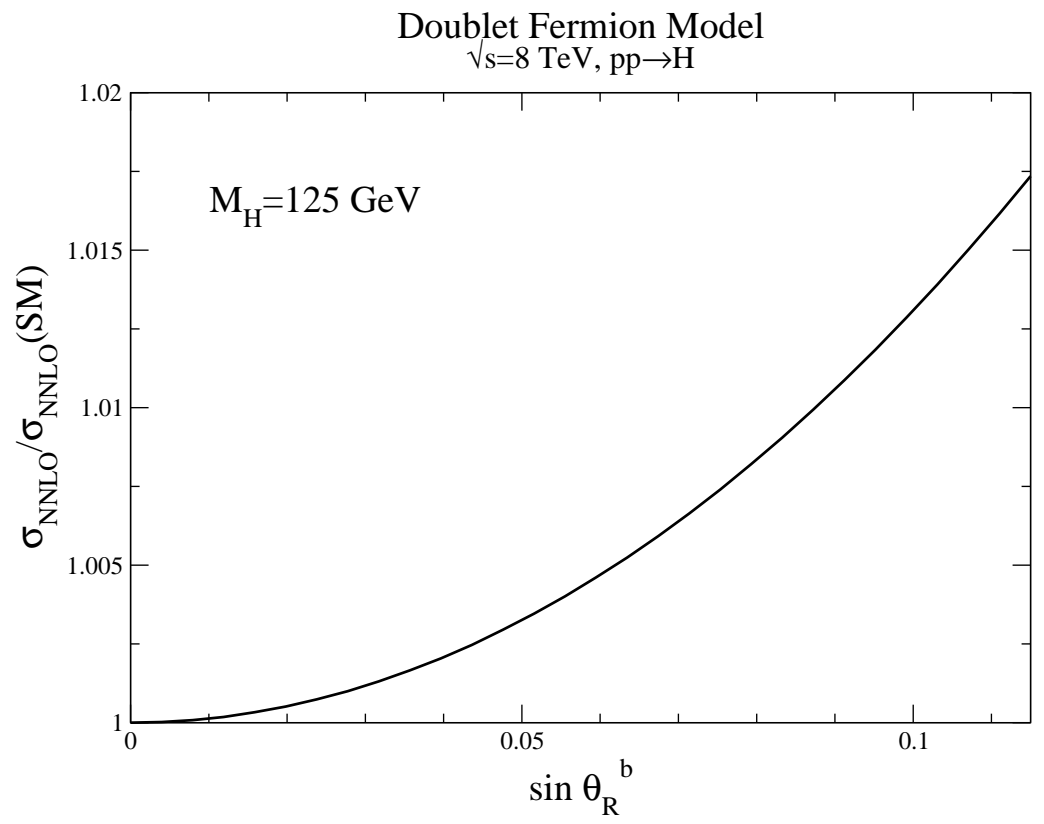

FIG. 15: The ratio of the NNLO Higgs cross section in the top partner doublet model normalized to the Standard Model prediction as a function of $\sin \theta_{R}^{b}$ for $M_{H}=125 \mathrm{GeV}, \sqrt{s}=8 \mathrm{TeV}$, and $M_{T}=M_{B}=1 \mathrm{TeV}$. 
the gluon fusion production of the Higgs,

$$
\begin{aligned}
\frac{\sigma_{\text {Doublet }}}{\sigma_{S M}} \simeq & \left(1+\sin ^{2} \theta_{R}^{b}\right)\left[1+\sin ^{2} \theta_{R}^{b}-\frac{7}{60} \frac{M_{H}^{2}}{m_{t}^{2}}\left(\frac{2 r-1}{r} \sin ^{2} \theta_{R}^{b}\right.\right. \\
& \left.\left.+\frac{2 \delta}{M_{T}}-\frac{2 \delta}{M_{T}} \frac{r+1}{r} \sin ^{2} \theta_{R}^{b}\right)\right] .
\end{aligned}
$$

From the fits to $A_{b}$ and $R_{b}$, the maximum value of $\sin \theta_{R}^{b}$ is restricted to be 0.115 , which implies

$$
\frac{\sigma_{\text {Doublet }}}{\sigma_{S M}} \lesssim\left(1+\sin ^{2} \theta_{R}^{b}\right)^{2} \simeq 1.03 .
$$

Similarly, the deviations from the Standard Model in the Higgs decays to $\gamma \gamma, Z \gamma$ and $g g$ and in $H \rightarrow b \bar{b}$, which is affected at tree level, are not observable due to the small mixings and mass splitting allowed.

\section{CONCLUSIONS}

We have considered the effects on the gluon fusion Higgs boson production at NNLO from heavy vector quarks of charge $2 / 3$ and $-1 / 3$. Since the new quarks are vector-like, their couplings to the Higgs boson are suppressed by mixing angles relative to the Standard Model Yukawa couplings. These mixing angles are restricted to be small by precision electroweak measurements. The most stringent bounds come from the oblique parameters for a vector singlet top-partner, and from $A_{b}$ and $R_{b}$ for an extension of the Standard Model with an additional vector doublet. Because of the small mixing angles allowed, in these models the Higgs boson production rate as well as its decay branching ratios are essentially those of the Standard Model. The scenarios we have presented will be extremely difficult to disentangle from the Standard Model without the observation of direct production of the heavy fermions. Vector doublet fermions with a non-standard hypercharge assignment are less restricted by precision electroweak measurements [36] and the mixing angles between the $t-b$ sector and the new fermion sector can be larger than in the cases we considered. However, even in this case, the low energy theorems for Higgs production require that the Higgs cross section approach the Standard Model result for large fermion masses.

If a Higgs boson is found at the LHC, attention will turn to understanding its properties. By performing global fits to the measured rates, information can be gleaned from the various cross section times branching ratio channels. For a light Higgs boson, it is likely that the 
dominant production channel will be gluon fusion, even in models with new physics. In this case, the rates are sensitive not only to a rescaling of the Standard Model couplings, but also to the effects of new particles which couple to the Higgs boson and contribute to the decay rates. Numerous preliminary attempts have been made to use current LHC data to discern differences from the Standard Model [81 90]. Our scenario with vector fermions demonstrates the difficulty of these indirect determinations of new physics - it is (un)fortunately not difficult to construct models which give Higgs signals indistinguishable from the Standard Model.

\section{Acknowledgements}

We would like to thank G. Panico for useful discussions. This work is supported by the United States Department of Energy under Grant DE-AC02-98CH10886.

\section{Appendix: Two Point Function for Arbitrary Fermion Coupling}

The contributions to the gauge boson two point functions from fermion loops parametrized by the interaction

$$
\mathcal{L}=\bar{f}_{1}\left(C_{L X}^{f_{1} f_{2}} P_{L}+C_{R X}^{f_{1} f_{2}} P_{R}\right) \gamma_{\mu} f_{2} V^{\mu}
$$

for $V=W, Z, \gamma$ are [21, 67]

$$
\begin{aligned}
\Pi_{X Y}= & -\frac{N_{c}}{16 \pi^{2}}\left\{\frac { 2 } { 3 } ( C _ { L X } ^ { f _ { 1 } f _ { 2 } } C _ { L Y } ^ { f _ { 1 } f _ { 2 } } + C _ { R X } ^ { f _ { 1 } f _ { 2 } } C _ { R Y } ^ { f _ { 1 } f _ { 2 } } ) \left[m_{1}^{2}+m_{2}^{2}-\frac{p^{2}}{3}-\left(A_{0}\left(m_{1}\right)+A_{0}\left(m_{2}\right)\right)\right.\right. \\
& \left.+\frac{m_{1}^{2}-m_{2}^{2}}{2 p^{2}}\left(A_{0}\left(m_{1}\right)-A_{0}\left(m_{2}\right)\right)+\frac{2 p^{4}-p^{2}\left(m_{1}^{2}+m_{2}^{2}\right)-\left(m_{1}^{2}-m_{2}^{2}\right)^{2}}{2 p^{2}} B_{0}\left(m_{1}, m_{2}, p^{2}\right)\right] \\
& \left.+2 m_{1} m_{2}\left(C_{L X}^{f_{1} f_{2}} C_{R Y}^{f_{1} f_{2}}+C_{R X}^{f_{1} f_{2}} C_{L Y}^{f_{1} f_{2}}\right) B_{0}\left(m_{1}, m_{2}, p^{2}\right)\right\}
\end{aligned}
$$

where

$$
\begin{aligned}
A_{0}(m) & =\left(\frac{4 \pi \mu^{2}}{m^{2}}\right)^{\epsilon} \Gamma(1+\epsilon)\left(\frac{1}{\epsilon}+1\right) m^{2} \\
B_{0}\left(m_{1}, m_{2}, p^{2}\right) & =\left(\frac{4 \pi \mu^{2}}{m_{2}^{2}}\right)^{\epsilon} \Gamma(1+\epsilon)\left[\frac{1}{\epsilon}-f_{1}\left(m_{1}, m_{2}, p^{2}\right)\right]
\end{aligned}
$$

and

$$
f_{1}\left(m_{1}, m_{2}, p^{2}\right)=\int_{0}^{1} d x \log \left(x+\frac{m_{1}^{2}(1-x)-p^{2} x(1-x)}{m_{2}^{2}}\right)
$$


[1] A.B. Arbuzov, M. Awramik, M. Czakon, A. Freitas, M.W. Grunewald, et al. ZFITTER: A Semi-analytical program for fermion pair production in $e^{+} e^{-}$annihilation, from version 6.21 to version 6.42. Comput.Phys.Commun., 174:728-758, 2006.

[2] Henning Flacher et al. Revisiting the Global Electroweak Fit of the Standard Model and Beyond with Gfitter. Eur. Phys. J., C60:543-583, 2009.

[3] Serguei Chatrchyan et al. Combined results of searches for the standard model Higgs boson in pp collisions at $\sqrt{s}=7 \mathrm{TeV}$. Phys.Lett., B710:26-48, 2012.

[4] Georges Aad et al. Combined search for the Standard Model Higgs boson using up to 4.9 fb-1 of pp collision data at $\sqrt{s}=7 \mathrm{TeV}$ with the ATLAS detector at the LHC. Phys.Lett., B710:49-66, 2012.

[5] Charalampos Anastasiou, Kirill Melnikov, and Frank Petriello. Higgs boson production at hadron colliders: Differential cross sections through next-to-next-to-leading order. Phys. Rev. Lett., 93:262002, 2004.

[6] Robert V. Harlander and William B. Kilgore. Next-to-next-to-leading order Higgs production at hadron colliders. Phys. Rev. Lett., 88:201801, 2002.

[7] LHC Higgs Cross Section Working Group, S. Dittmaier, C. Mariotti, G. Passarino, and R. Tanaka (Eds.). Handbook of LHC Higgs Cross Sections: 1. Inclusive Observables. CERN2011-002, CERN, Geneva, 2011.

[8] Charalampos Anastasiou, Stephan Buehler, Franz Herzog, and Achilleas Lazopoulos. Total cross-section for Higgs boson hadroproduction with anomalous Standard Model interactions. JHEP, 12:058, 2011.

[9] Daniel de Florian and Massimiliano Grazzini. Higgs production through gluon fusion: Updated cross sections at the Tevatron and the LHC. Phys.Lett., B674:291-294, 2009.

[10] Charalampos Anastasiou, Radja Boughezal, and Frank Petriello. Mixed QCD-electroweak corrections to Higgs boson production in gluon fusion. JHEP, 0904:003, 2009.

[11] Charalampos Anastasiou, Radja Boughezal, and Elisabetta Furlan. The NNLO gluon fusion Higgs production cross-section with many heavy quarks. JHEP, 06:101, 2010.

[12] Charalampos Anastasiou, Stephan Buehler, Elisabetta Furlan, Franz Herzog, and Achilleas 
Lazopoulos. Higgs production cross-section in a Standard Model with four generations at the LHC. Phys. Lett., B702:224-227, 2011.

[13] Elisabetta Furlan. Gluon-fusion Higgs production at NNLO for a non-standard Higgs sector. JHEP, 10:115, 2011.

[14] Graham D. Kribs, Tilman Plehn, Michael Spannowsky, and Timothy M.P. Tait. Four generations and Higgs physics. Phys.Rev., D76:075016, 2007.

[15] Otto Eberhardt, Alexander Lenz, and Jurgen Rohrwild. Less space for a new family of fermions. Phys.Rev., D82:095006, 2010.

[16] The CMS collaboration. Combined results of searches for a Higgs boson in the context of the Standard Model and Beyond-Standard Models.

[17] N. Arkani-Hamed, A.G. Cohen, E. Katz, and A.E. Nelson. The Littlest Higgs. JHEP, 0207:034, 2002.

[18] Ian Low, Witold Skiba, and David Tucker-Smith. Little Higgses from an antisymmetric condensate. Phys.Rev., D66:072001, 2002.

[19] Maxim Perelstein, Michael E. Peskin, and Aaron Pierce. Top quarks and electroweak symmetry breaking in little Higgs models. Phys.Rev., D69:075002, 2004.

[20] Spencer Chang and Jay G. Wacker. Little Higgs and custodial SU(2). Phys.Rev., D69:035002, 2004 .

[21] $\mathrm{Mu}-\mathrm{Chun}$ Chen and Sally Dawson. One loop radiative corrections to the rho parameter in the littlest Higgs model. Phys.Rev., D70:015003, 2004.

[22] Jay Hubisz, Patrick Meade, Andrew Noble, and Maxim Perelstein. Electroweak precision constraints on the littlest Higgs model with T parity. JHEP, 0601:135, 2006.

[23] Tao Han, Heather E. Logan, and Lian-Tao Wang. Smoking-gun signatures of little Higgs models. JHEP, 0601:099, 2006.

[24] Christopher T. Hill. Topcolor: Top quark condensation in a gauge extension of the standard model. Phys.Lett., B266:419-424, 1991.

[25] Christopher T. Hill and Elizabeth H. Simmons. Strong dynamics and electroweak symmetry breaking. Phys.Rept., 381:235-402, 2003.

[26] Bogdan A. Dobrescu and Christopher T. Hill. Electroweak symmetry breaking via top condensation seesaw. Phys.Rev.Lett., 81:2634-2637, 1998.

[27] R. Sekhar Chivukula, Bogdan A. Dobrescu, Howard Georgi, and Christopher T. Hill. Top 
quark seesaw theory of electroweak symmetry breaking. Phys.Rev., D59:075003, 1999.

[28] Hong-Jian He, Timothy M.P. Tait, and C.P. Yuan. New top flavor models with seesaw mechanism. Phys.Rev., D62:011702, 2000.

[29] Hidenori S. Fukano and Kimmo Tuominen. A hybrid $4^{\text {th }}$ generation: Technicolor with topseesaw. 2012.

[30] Roberto Contino, Leandro Da Rold, and Alex Pomarol. Light custodians in natural composite Higgs models. Phys.Rev., D75:055014, 2007.

[31] Marcela S. Carena, Eduardo Ponton, Jose Santiago, and Carlos E.M. Wagner. Light Kaluza Klein States in Randall-Sundrum Models with Custodial SU(2). Nucl.Phys., B759:202-227, 2006.

[32] Riccardo Barbieri, B. Bellazzini, Vyacheslav S. Rychkov, and Alvise Varagnolo. The Higgs boson from an extended symmetry. Phys.Rev., D76:115008, 2007.

[33] Oleksii Matsedonskyi, Giuliano Panico, and Andrea Wulzer. Light Top Partners for a Light Composite Higgs. 2012.

[34] P. Bamert, C.P. Burgess, James M. Cline, David London, and E. Nardi. R(b) and new physics: A Comprehensive analysis. Phys.Rev., D54:4275-4300, 1996.

[35] D. Comelli and Joao P. Silva. Decoupling or nondecoupling: Is that the R(b) question? Phys.Rev., D54:1176-1181, 1996.

[36] Giacomo Cacciapaglia, Aldo Deandrea, Daisuke Harada, and Yasuhiro Okada. Bounds and Decays of New Heavy Vector-like Top Partners. JHEP, 1011:159, 2010.

[37] K. Nakamura et al. Review of particle physics. J.Phys.G, G37:075021, 2010.

[38] A.A. Akhundov, D. Yu. Bardin, and T. Riemann. Electroweak One Loop Corrections to the Decay of the Neutral Vector Boson. Nucl.Phys., B276:1, 1986.

[39] J. Bernabeu, A. Pich, and A. Santamaria. $\Gamma(Z \rightarrow b \bar{b})$ : A Signature of Hard Mass Terms for a Heavy Top. Phys.Lett., B200:569, 1988.

[40] Wim Beenakker and Wolfgang Hollik. The Width of the Z Boson. Z.Phys., C40:141, 1988.

[41] C.P. Burgess, Stephen Godfrey, Heinz Konig, David London, and Ivan Maksymyk. Model independent global constraints on new physics. Phys.Rev., D49:6115-6147, 1994.

[42] Howard E. Haber and Heather E. Logan. Radiative corrections to the Z b anti-b vertex and constraints on extended Higgs sectors. Phys.Rev., D62:015011, 2000.

[43] Precision electroweak measurements on the $Z$ resonance. Phys.Rept., 427:257-454, 2006. 302 
pages, v2: minor corrections and updates of references. Accepted for publication by Physics Reports, v3: further small corrections and journal version Report-no: CERN-PH-EP/2005041, SLAC-R-774.

[44] Michael E. Peskin and Tatsu Takeuchi. Estimation of oblique electroweak corrections. Phys.Rev., D46:381-409, 1992.

[45] Guido Altarelli and Riccardo Barbieri. Vacuum polarization effects of new physics on electroweak processes. Phys. Lett., B253:161-167, 1991.

[46] Riccardo Barbieri, Alex Pomarol, Riccardo Rattazzi, and Alessandro Strumia. Electroweak symmetry breaking after LEP-1 and LEP-2. Nucl.Phys., B703:127-146, 2004.

[47] M. Baak et al. Updated Status of the Global Electroweak Fit and Constraints on New Physics. 2011.

[48] W. Hollik. Electroweak radiative corrections. 1993.

[49] Mu-Chun Chen, Sally Dawson, and C.B. Jackson. Higgs Triplets, Decoupling, and Precision Measurements. Phys.Rev., D78:093001, 2008.

[50] Georges Aad et al. Search for pair production of a heavy quark decaying to a W boson and a b quark in the lepton + jets channel with the ATLAS detector. 2012.

[51] Georges Aad et al. Search for pair production of a new quark that decays to a Z boson and a bottom quark with the ATLAS detector. Phys.Rev.Lett., 2012. Long author list - awaiting processing.

[52] Serguei Chatrchyan et al. Search for heavy, top-like quark pair production in the dilepton final state in pp collisions at $\sqrt{s}=7 \mathrm{TeV}$. 2012. Submitted to Physics Letters B.

[53] Serguei Chatrchyan et al. Search for heavy bottom-like quarks in 4.9 inverse femtobarns of pp collisions at $\sqrt{s}=7 \mathrm{TeV} .2012$.

[54] Serguei Chatrchyan et al. Search for a Vector-like Quark with Charge $2 / 3$ in t + Z Events from pp Collisions at $\sqrt{s}=7 \mathrm{TeV}$. Phys.Rev.Lett., 107:271802, 2011.

[55] J.A. Aguilar-Saavedra. Identifying top partners at LHC. JHEP, 0911:030, 2009.

[56] Christian J. Flacco, Daniel Whiteson, and Matthew Kelly. Fourth generation quark mass limits in CKM-element space. 2011.

[57] Giacomo Cacciapaglia, Aldo Deandrea, Luca Panizzi, Naveen Gaur, Daisuke Harada, et al. Heavy Vector-like Top Partners at the LHC and flavour constraints. JHEP, 1203:070, 2012.

[58] Keisuke Harigaya, Shigeki Matsumoto, Mihoko M. Nojiri, and Kohsaku Tobioka. Search for 
the Top Partner at the LHC using Multi-b-Jet Channels. 2012.

[59] Joshua Berger, Jay Hubisz, and Maxim Perelstein. A Fermionic Top Partner: Naturalness and the LHC. 2012.

[60] J.A. Aguilar-Saavedra. Effects of mixing with quark singlets. Phys.Rev., D67:035003, 2003.

[61] Monika Blanke, Andrzej J. Buras, Anton Poschenrieder, Cecilia Tarantino, Selma Uhlig, et al. Particle-Antiparticle Mixing, $\varepsilon_{K}, \Delta \Gamma_{q}, A_{\mathrm{SL}}^{q}, A_{\mathrm{CP}}\left(B_{d} \rightarrow \psi K_{S}\right), A_{\mathrm{CP}}\left(B_{s} \rightarrow \psi \phi\right)$ and $B \rightarrow$ $X_{s, d} \gamma$ in the Littlest Higgs Model with T-Parity. JHEP, 0612:003, 2006.

[62] L. Lavoura and Joao P. Silva. Bounds on the mixing of the down type quarks with vector like singlet quarks. Phys.Rev., D47:1117-1126, 1993.

[63] Marko B. Popovic and Elizabeth H. Simmons. Weak singlet fermions: Models and constraints. Phys.Rev., D62:035002, 2000.

[64] Tevatron Electroweak Working Group. Combination of CDF and D0 Results on the Mass of the Top Quark. 2009. FERMILAB-TM-2427-E, TEVEWWG/top 2009/03, CDF Note 9717, D0-NOTE-5899 15 pages, 1 figure.

[65] Michael S. Chanowitz, M. A. Furman, and I. Hinchliffe. Weak Interactions of Ultraheavy Fermions. 2. Nucl. Phys., B153:402, 1979.

[66] S. Dawson and P. Jaiswal. Four Generations, Higgs Physics, and the MSSM. Phys.Rev., D82:073017, 2010.

[67] Fred Jegerlehner. Renormalizing the standard model. Conf.Proc., C900603:476-590, 1990.

[68] G. Cynolter and E. Lendvai. Electroweak Precision Constraints on Vector-like Fermions. Eur.Phys.J., C58:463-469, 2008.

[69] Nobuhiro Maekawa. Electroweak symmetry breaking by vector - like fermions' condensation with small S and T parameters. Phys.Rev., D52:1684-1692, 1995.

[70] Hong-Jian He, Christopher T. Hill, and Timothy M.P. Tait. Top quark seesaw, vacuum structure and electroweak precision constraints. Phys.Rev., D65:055006, 2002.

[71] Csaba Csaki, Jay Hubisz, Graham D. Kribs, Patrick Meade, and John Terning. Variations of little Higgs models and their electroweak constraints. Phys.Rev., D68:035009, 2003.

[72] Yang Bai, JiJi Fan, and JoAnne L. Hewett. Hiding a Heavy Higgs Boson at the 7 TeV LHC. 2011.

[73] P. Bamert. R(b) and heavy quark mixing. 1996.

[74] Charalampos Anastasiou, Elisabetta Furlan, and Jose Santiago. Realistic Composite Higgs 
Models. Phys.Rev., D79:075003, 2009.

[75] Marcela S. Carena, Eduardo Ponton, Jose Santiago, and C.E.M. Wagner. Electroweak constraints on warped models with custodial symmetry. Phys.Rev., D76:035006, 2007.

[76] L. Lavoura and Joao P. Silva. The Oblique corrections from vector - like singlet and doublet quarks. Phys.Rev., D47:2046-2057, 1993.

[77] Frank Wilczek. Decays of Heavy Vector Mesons Into Higgs Particles. Phys.Rev.Lett., 39:1304, 1977.

[78] John R. Ellis, M.K. Gaillard, Dimitri V. Nanopoulos, and Christopher T. Sachrajda. Is the Mass of the Higgs Boson About 10-GeV? Phys.Lett., B83:339, 1979.

[79] H.M. Georgi, S.L. Glashow, M.E. Machacek, and Dimitri V. Nanopoulos. Higgs Bosons from Two Gluon Annihilation in Proton Proton Collisions. Phys.Rev.Lett., 40:692, 1978.

[80] Adam Falkowski. Pseudo-goldstone Higgs production via gluon fusion. Phys.Rev., D77:055018, 2008.

[81] Ian Low, Riccardo Rattazzi, and Alessandro Vichi. Theoretical Constraints on the Higgs Effective Couplings. JHEP, 1004:126, 2010.

[82] Bogdan A. Dobrescu, Graham D. Kribs, and Adam Martin. Higgs Underproduction at the LHC. Phys.Rev., D85:074031, 2012. 8 pages, 9 figures. Minor improvements made and references added.

[83] Christoph Englert, Tilman Plehn, Michael Rauch, Dirk Zerwas, and Peter M. Zerwas. LHC: Standard Higgs and Hidden Higgs. Phys.Lett., B707:512-516, 2012. 7 pages, 3 figures/ references added/ final version accepted for publication in PLB.

[84] J.R. Espinosa, C. Grojean, and M. Muhlleitner. Composite Higgs under LHC Experimental Scrutiny. 2012. 6 pages. Contribution to the proceedings of Hadron Collider Physics Symposium 2011, Paris Nov. 14-18.

[85] Dean Carmi, Adam Falkowski, Eric Kuflik, and Tomer Volansky. Interpreting LHC Higgs Results from Natural New Physics Perspective. 2012.

[86] Aleksandr Azatov, Roberto Contino, and Jamison Galloway. Model-Independent Bounds on a Light Higgs. JHEP, 1204:127, 2012.

[87] J.R. Espinosa, Christophe Grojean, M. Muhlleitner, and Michael Trott. Fingerprinting Higgs Suspects at the LHC. 2012.

[88] Pier Paolo Giardino, Kristjan Kannike, Martti Raidal, and Alessandro Strumia. Reconstruct- 
ing Higgs boson properties from the LHC and Tevatron data. 2012. 15 pages, 5 figures, references added, discussion clarified (e.g. fit to invisible width), fit to radion added.

[89] John Ellis and Tevong You. Global Analysis of Experimental Constraints on a Possible HiggsLike Particle with Mass 125 GeV. 2012.

[90] Markus Klute, Remi Lafaye, Tilman Plehn, Michael Rauch, and Dirk Zerwas. Measuring Higgs Couplings from LHC Data. 2012. 